\title{
ON THE PICARD-LEFSCHETZ TRANSFORMATION FOR ALGEBRAIC MANIFOLDS ACQUIRING GENERAL SINGULARITIES
}

\author{
BY
}

\section{ALAN LANDMAN}

\begin{abstract}
We consider a holomorphic family $\left\{V_{t}\right\}_{t \in D}$ of projective algebraic varieties $V_{t}$ parametrized by the unit disc $D=\{t \in C:|t|<1\}$ and where $V_{t}$ is smooth for $t \neq 0$ but $V_{0}$ may have arbitrary singularities. Displacement of cycles around a path $t=t_{0} e^{i \theta}(0 \leq \theta \leq 2 \pi)$ leads to the Picard-Lefschetz transformation $T: H_{*}\left(V_{t_{0}}, \mathrm{Z}\right) \rightarrow H_{*}\left(V_{t_{0}}, \mathrm{Z}\right)$ on the homology of a smooth $V_{t_{0}}$. We prove that the eigenvalues of $T$ are roots of unity and obtain an estimate on the elementary divisors of $T$. Moreover, we give a global inductive procedure for calculating $T$ in specific examples, several of which are worked out to illustrate the method.
\end{abstract}

\section{Introduction.}

A. Let $W$ and $W^{\prime}$ be algebraic varieties and $f: W \rightarrow W^{\prime}$ a rational map. For $t \in W^{\prime}$, let $V_{t}=f^{-1}(t)$ be the corresponding fibre.

In general, there exists an algebraic subset $S$ of $W^{\prime}$ such that $W-f^{-1}(S)$ is a fibre space over $W^{\prime}-S$ via $f$. This induces a representation of the fundamental group $\pi_{1}\left(W^{\prime}-S, t\right)$ as a group of homeomorphisms of $V_{t}$. Hence, $\pi_{1}\left(W^{\prime}-S, t\right)$ acts as a group of linear transformations on the homology $H_{*}\left(V_{t}\right)$.

This leads to the following problem:

Given a loop in $W^{\prime}-S$, describe the corresponding transformation.

B. As it stands, this problem is too general. We will make the following simplifying assumptions:

(1.1) $W$ is projective and nonsingular.

(1.2) $W^{\prime}$ is a projective, nonsingular curve $C$. This means that the fibres $V_{t}$ are divisors on $W$, i.e., their components may have multiplicities greater than 1.

Assumption (1.2) is useful because in many cases $\pi_{1}\left(W^{\prime}-S, t\right)$ is generated by $\pi_{1}(C-C \cap S, t)$, where $C$ is a general curve in $W^{\prime}$ containing $t$, e.g., a plane section relative to some projective embedding of $W^{\prime}$. When $W^{\prime}$ is a projective space, this was shown by Zariski [8].

Received by the editors November 25, 1971.

AMS (MOS) subject clas sifications (1970). Primary 14D05.

Key words and phrases. Picard-Lefschetz transformation (P-L transformation), vanishing cycle, vanishing cone, general pencil of hyperplane sections, branch function, branch curve, normal crossings, monodromy theorem, Gauss-Manin connection, Hodge decomposition. 
(1.3) $f$ is defined on all of $W$, i.e., there is no base locus. (This is not essential and will be weakened later on.)

With the se assumptions, Bertini's theorem tells us that all the $V_{t}$ are nonsingular except for those $t$ belonging to a certain finite subset of $C$. These exceptional points form the set $S$ mentioned above.

(1.4) The loop $g$ in which we are interested is a simple closed curve containing just one point $p$ of $S$.

The action on homology induced by $g$ depends only on the nature of the singularities of $V_{p}$. When $V_{p}$ has only ordinary double points, Picard [6] and Lefschetz [4] gave a formula for expressing the transformation. This is why we call the action in more general situations the Picard-Lefschetz transformation (or just the P-L transformation).

(1.5) $V_{p}$ has only normal crossings, i.e., its underlying algebraic set is the union of nonsingular hypersurfaces of $W$ meeting transversally (cf. part 9B).

C. We begin by describing Lefschetz's method for obtaining the homology of an algebraic variety by means of hyperplane sections. This is applied to each fibre $V_{t}$, for $t$ near $p$, to yield the following results:

(1.6) Theorem I. The eigenvalues of the P.L transformation are roots of unity.

More precisely, we have

(1.7) Theorem I'. Let $m_{1}, \cdots, m_{r}$ be the multiplicities of the components of $V_{p}$. Then each eigenvalue of the P-L transformation is an $m_{i}$ th root of unity for some $i=1, \cdots, r$.

(1.8) Theorem II. The elementary divisors of the P-L transformation are all of degree $\leq \operatorname{dim} W$.

More precisely, we have

(1.9) Theorem II'. Let $s$ be the maximum number of components of $V_{p}$ which bave a point in common. Then the elementary divisors of the P.L transformation are all of degree $\leq s$.

We conclude by examining the P-L transformation in some specific cases.

2. The 0-dimensional case.

A. When $W$ itself is a curve, so that the $V_{t}$ are 0 -dimensional, the P-L transformation can be described through the classical theory of algebraic functions of one variable.

Let $x \in W$ and let $z$ be a local coordinate at $x$ in $W$. Let $t$ be a local coordinate at $f(x)$ in $C$, so that near $x$ the function $f$ is given by 


$$
t=f(z)=c_{1} z^{m}+c_{2} z^{m+1}+\cdots
$$

where $c_{1} \neq 0$ and $m$ is the multiplicity of $x$ as a component of $V_{0}=f^{-1}(f(x))$.

The power series (2.1) can be inverted to yield the Puiseux series

$$
z=\left(1 / c_{1}\right) t^{1 / m}+\cdots
$$

which is a power series in $t^{1 / m}$. Hence, when $t$ is near $0, V_{t}$ consists of $m$ points near $x$ and these points permute cyclically as $t$ makes one circuit about 0 .

Note that the fibre $V_{0}$ consists of normal crossings and the P-L transformation satisfies the theorems stated in part $1 C$.

B. The homology of the curve $W$ can be determined in a classical way by means of the pencil $V_{t}$.

For simplicity, let us assume that the parameter curve $C$ is actually the projective line $P^{1}$. There will be a finite set $R$ in $W$ at which $f$ ramifies, i.e., points where the $m$ of $(2.1)$ is $>1$. Let $S=f(R)$. Then $t \in S$ if and only if $V_{t}$ is singular, i.e., $V_{t}=m_{1} x_{1}+\cdots+m_{r} x_{r}$, where the $x_{i}$ are distinct points of $W$, the $m_{i}$ are positive integers and at least one $m_{i}$ is $>1$. The behavior of the points of $W$ near the $x_{i}$ was mentioned above.

From the continuity of algebraic functions, we deduce the following:

(2.3) $f: W-f^{-1}(S) \rightarrow P^{1}-S$ is a fibre space with fibre any nonsingular $V_{t}$.

(2.4) If $g:[0,1] \rightarrow P^{1}$ is a continuous map of the unit interval into $P^{1}$ such that $g[0,1) \subseteq P^{1}-S$ and if $y \in f^{-1}(g(0))$, then there is a unique map $\bar{g}$ : $[0,1] \rightarrow W$ such that $f \circ \bar{g}=g$ and $\bar{g}(0)=y$.

(2.4) implies that there is a continuous map $T_{g}$ from $f^{-1}(g(0))$ to $f^{-1}(g(1))$. In particular, $\pi_{1}\left(P^{1}-S, p\right)$ acts on $H_{0}\left(f^{-1}(p)\right)$.

(2.5) In case $g(1) \in S$ and $f^{-1}(g(1))=m_{1} x_{1}+\cdots+m_{r} x_{r}$, then $T_{g}$ is onto and $T_{g}^{-1}\left(x_{i}\right)$ consists of $m_{i}$ points.

This leads us to two important concepts. The first is that of vanishing cycle (along $g$ ), which is just an element $d$ of $H_{0}\left(f^{-1}(g(0))\right)$ such that $T_{g}(d)=0$ in $H_{0}\left(f^{-1}(g(1))\right)$. In the case at hand, the vanishing cycles are generated by those of the form $y_{1}-y_{2}$, where $T_{g}\left(y_{1}\right)=T_{g}\left(y_{2}\right)$.

Next, we have the concept of the cylinder generated by a cycle $d$ in $H_{0}\left(f^{-1}(g(0))\right)$. We simply keep track of the cycle in each fibre over $g[0,1]$. This cylinder, denoted $D(d, g)$, is a 1-chain which, with appropriate orientation, has boundary $d-T_{g}(d)$.

In the special case where $d$ vanishes along $g, \partial D(d, g)=d$ and we can speak of the cone generated by $d$. It is an element of the relative group $H_{1}\left(W, f^{-1}(g(0))\right)$.

(2.6) Pick a base-point $p$ in $P^{1}$ and let $\left\{g_{i}\right\}$ be simple paths from $p$ to the points of $S$ meeting only at $p$. Then $H_{1}\left(W, V_{p}\right)$ is generated by the cones over the $g_{i}$. 
In particular, if we assume that the singular fibres are as simple as possible, viz., of the form $2 x_{1}+x_{2}+\cdots+x_{r}$ (the $x_{i}$ being distinct points of $W$ ), then there is exactly one vanishing cycle $d_{i}$ for each $g_{i} \cdot d_{i}$ will be of the form $u_{i}-$ $v_{i}$, where $u_{i}, v_{i}$ are points in $V_{p}$. Hence,

(2.7) $\left(d_{i}, d_{i}\right)=2$, where $($,$) is the Kronecker intersection pairing in$ $H_{0}\left(V_{p}\right)$.

If we replace $g_{i}$ by the corresponding loop around its endpoint in $S$, this loop induces the identity transformation on $V_{p}$ except that $u_{i}$ and $v_{i}$ are interchanged. This can be expressed as

(2.8) The P-L transformation is given by the formula $b \rightarrow b-\left(b, d_{i}\right) d_{i}$, for $b \in H_{0}\left(V_{p}\right)$.

3. The Lefschetz theorems.

A. The P-L transformation we seek can be considered a local problem. That is, we have a pencil $V_{t}$ on $W$ and we wish to examine its (homology) behavior near one (singular) member. This was described in part $2 \mathrm{~A}$ when $W$ is a curve.

But there is also a global theory analogous to (2.6) in which the pencil determines the homology of the ambient $W$. This was developed by Lefschetz [4] and is described below. Using his global construction of homology in a given dimension (that of the $V_{t}$ ), we can determine the (local) P-L transformation in the dimension one higher.

B. At this point we mention some topological facts which generalize (2.3) and (2.4).

We have our pencil of divisors $V_{t}$ given as the fibres under the rational map $f: W \rightarrow C$, where $C$ is a curve. If $\operatorname{dim} W>1$, the first new phenomenon we meet is that $f$ may not be defined on all of $W$, i.e., there may be a base locus $B$ of codimension 2 in $W$ which is common to all the $V_{t}$. For the purposes of the present section, this will be more of a help than a hindrance (see part 11 for more on pencils with base).

Each fibre $V_{t}$ has a tubular neighborhood in $W$, i.e., a neighborhood of which it is a deformation retract, and if $t^{\prime}$ is near enough to $t$, then $V_{t^{\prime}}$ will be in that neighborhood. Hence, there is an associated map $g_{t^{\prime}{ }_{t}}: V_{t^{\prime}} \rightarrow V_{t}$ unique up to homotopy. If $V_{t}$ is nonsingular, then $V_{t^{\prime}}$ will be a cross-section of the tubular neighborhood and $g_{t^{\prime} t}$ will be a homeomorphism.

We assume that the general $V_{t}$ is nonsingular and irreducible. Then there is a finite subset $S \subseteq C$ such that $V_{t}$ is singular if and only if $t \in S$. Using the tubular neighborhoods, $W-f^{-1}(S)-B$ becomes a fibre bundle over $C-S$ via $f$ (see Wallace [7]).

Hence, if $g:[0,1] \rightarrow C$ is a path such that $g[0,1) \subseteq C-S$, then $V_{g(1)}$ is a deformation retract of $\bigcup_{v \in[0,1]} V_{g(v)}$ and if $d$ is a subset or cycle in $V_{g(0)}$, 
we will have a corresponding cylinder unique up to homotopy. The cylinders over homotopic paths are homologous. As in part $2 \mathrm{~B}$, we have the notions of vanishing cycle, cone, etc.

C. Lefschetz first finds the P-L transformation when the singular fibre $V_{0}$ has an ordinary double point off the base.

$A_{n}$ ordinary double point can be described in several equivalent ways:

$x$ is an ordinary double point for $V_{0}$ if

(3.1) the tangent cone to $V_{0}$ at $x$ is of degree 2 without multiple generators, or

(3.2) if $z_{1}, \cdots, z_{n}$ are local coordinates at $x$ in $W$, then $V_{0}$ is given locally by an equation $H\left(z_{1}, \cdots, z_{n}\right)=0$, where

(1) $\partial H /\left.\partial z_{i}\right|_{x}=0$ for all $i$, that is, $x$ is singular for $V_{0}$,

(2) $\left|\partial^{2} H / \partial z_{i} \partial z_{j}\right|_{x} \neq 0$, that is, the partials of $H$ form a local coordinate system at $x$,

or,

(3.3) local coordinates $z_{1}, \cdots, z_{n}$ can be found so that $V_{0}$ is given by $z_{1}^{2}+\cdots+z_{n}^{2}=0$ (Fáry [1]).

Hence, if $t$ is a local coordinate at $f(x)$ in $C$, then the pencil can be given locally by $z_{1}^{2}+\cdots+z_{n}^{2}=t$. We then have

(3.4) The P-L transformation from $H_{i}\left(V_{t}\right)$ to itself is the identity if $i \neq n-1$.

(3.5) If $d_{1}=\left\{z_{i}\right.$ real $\left.\mid z_{1}^{2}+\cdots+z_{n}^{2}=1\right\}$ and $d_{t}=t^{-1 / 2} d_{1}$, then $d_{t}$ is an $(n-1)$-sphere in $V_{t}(t \neq 0)$ representing a homology class in $H_{n-1}\left(V_{t}\right)$ such that $d_{t} \rightarrow 0$ as $t \rightarrow 0$, i.e., $d_{t}$ is a vanishing cycle on $V_{t}$.

(3.6) If $c \in H_{n-1}\left(V_{t}\right)$, then $c$ goes to $c+(-1)^{n(n+1) / 2}\left(c, d_{t}\right) d_{t}$ as $t$ goes around 0 .

(3.7) If $n$ is odd, $d_{t}$ goes to $-d_{t}$ and $\left(d_{t}, d_{t}\right)=(-1)^{(n-1) / 2} 2$; if $n$ is even, $d_{t}$ goes to $d_{t}$ and $\left(d_{t}, d_{t}\right)=0$.

D. Lefschetz's global theorems require that the pencil $V_{t}$ belong to a "sufficiently general" linear system $V$. According to Lefschetz, this means

(3.8) $\operatorname{dim}|V| \geq n$ and any $n$ members of $|V|$ have a point in common.

These properties, of course, are satisfied by any pencil on a curve, which fits in with the results of part 2. They are also satisfied by the system of hyperplane sections of $W$ (relative to some projective embedding), and this is the system we will be using (see part 4).

As we did before, we cut the parameter line by picking a base-point $p \notin S$ and tracing nonintersecting cuts $g_{i}$ from $p$ to the points of $S$. Then we have

(3.9) $H_{i}\left(W, V_{t}\right)=0, i \leq n-1=\operatorname{dim} V_{t}$ (for any $\left.V_{t}\right)$.

(3.10) $H_{n}\left(W, V_{p}\right)$ is generated by the cones over the $g_{i}$.

These hold regardless of the nature of the singular fibres. It is often 
convenient to assume that the singular $V_{t}$ have one ordinary double point off the base (see part $4 \mathrm{~A}$ for such a possibility), so that (3.6) tells us that there is just one cone for each $g_{i}$.

Let $D_{i}=D\left(d_{i}, g_{i}\right)$. What are the relations among the $D_{i}$ in $H_{n}\left(W, V_{p}\right)$ ? There is one obvious way to generate such relations:

Let $U=P^{1}-\bigcup_{i} g_{i}$. Then $U$ is homeomorphic to a disc and $f: W-$ $f^{-1}\left(\bigcup_{i} g_{i}\right) \rightarrow U$ is the trivial bundle. If $V_{t}-B$ is a typical fibre $(t \in U)$, pick a (compact or noncompact) cycle $c$ in it. (This is really the same as a compact cycle in $H_{n-1}\left(V_{t}, B\right)$.)

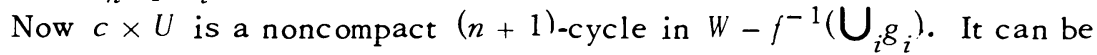
thought of as the "translates" of $c$ to the other fibres over $U$ (these are coherent, because the bundle is trivial). What is $\partial(c \times U)$ in $W$ ? It is the $n$-chain $\left(\partial_{c}\right) \times U+c \times(\partial U)$ (viewing $c$ now in $H_{n-1}\left(V_{t}, B\right)$ ).

$\left(\partial_{c}\right) \times U$ are the translates of $\partial_{c}$ in the fibres $V_{t}(t \in U)$, but since $\partial_{c} \subseteq B$ and $B$ is always left point-wise fixed under the various deformation retracts (part 3B), the collection of translates of $\partial_{c}$ consists of $\partial_{c}$ itself. But this is only $(n-1)$-dimensional and so does not figure effectively in $\partial(c \times U)$.

As for $c \times(\partial U)$ (or, at least, its $n$-dimensional pieces), observe that each $g_{i}$ contributes two edges to $\partial U$ in the sense that as $c$ approaches the same point $t$ of $g_{i}$ (except $p$ ) from opposite sides, the resulting cycles may not be homologous in $H_{n-1}\left(V_{t}\right)$. In fact, by (3.6), they differ by a multiple of $d_{i}$ (i.e., after $d_{i}$ has been put in $V_{t}$ via $g_{i}$ ). Hence, as $t$ traces $g_{i}$, c traces a certain multiple of $D_{i}$.

Hence, for some integers $n_{i}, \partial(c \times U)=n_{1} D_{1}+\cdots+$ something in $V_{p}(p$ being the only other part of $\partial U)$, i.e., we have a relation in $H_{n}\left(W, V_{p}\right)$. Then,

(3.11) All relations among the $D_{i}$ arise this way.

(3.12) If $c \in H_{n-1}\left(V_{t}, B\right)$ yields the trivial relation, then $\partial_{c}=0$ and $c$ is invariant under all the P-L transformations, i.e., $\left(c, d_{i}\right)=0$ for all $i$.

Hence,

(3.13) rank $H_{n}\left(W, V_{p}\right)=m-\operatorname{rank} H_{n-1}\left(V_{t}, B\right)+\operatorname{rank} H_{n-1}(W)$, where $m$ is the number of points in $S$ and $V_{t}$ is any nonsingular fibre.

E. Suppose now the $V_{t}$ are hyperplane sections and let $H$ be one of them (one can think of it as the "hyperplane at infinity"). Then $W-H$ and the $V_{t}-$ $H$ are affine varieties and we have (Fáry [1], Žižcenko [9])

(3.14) $H_{i}(W-H)=0, i>\operatorname{dim} W$ and similarly,

(3.15) $H_{i}\left(V_{t}-H\right)=0, i>\operatorname{dim} V_{t}$ for any $t$.

(3.16) $H_{i}\left(W-H, V_{t}-H\right)=0, i<n=\operatorname{dim} W$.

(3.17) $H_{n}\left(W-H, V_{t}-H\right)$ is generated by the $D_{i}$ and these are independent, i.e., $H_{n}\left(W-H, V_{t}-H\right) \simeq \mathbf{Z} \oplus \cdots \oplus \mathbf{Z}$ (m times). 


\section{Hyperplane sections.}

A. Parts $3 \mathrm{D}$ and $3 \mathrm{E}$ show how important hyperplane sections are for describing the homology of varieties. We now mention some facts about hyperplane sections that will be useful later on (see Wallace [7]).

If $V$ is a nonsingular irreducible variety in a projective space $P_{N}$, then the general hyperplane section of $V$ is nonsingular (Bertini's theorem). Hence, the collection of hyperplanes cutting singular sections on $V$ (or containing $V$ entirely) forms a proper irreducible subvariety $V^{*}$ of the dual space $P_{N}^{*} \cdot V^{*}$ is called the dual of $V$. We assume that $V$ is not just a linear subspace of $P_{N}$ so that $V^{*}$ is a hypersurface in $P_{N^{*}}^{*}$ Its degree $m$ is called the class of $V$.

A nonsingular point $H$ of $V^{*}$ corresponds to a section of $V$ with one ordinary double point (part 3C) and the tangent plane to $V^{*}$ at $H$ consists of those hyperplanes of $P_{N}$ which pass through that double point.

Now take a line $L$ in $P_{N}^{*}$ which is in general position with respect to $V^{*}$. $L$ just represents a pencil of hyperplanes in $P_{N}$. Since $L$ is general, it meets $V^{*}$ in $m$ distinct points. These points are nonsingular for $V^{*}$ and $L$ is not tangent to $V^{*}$ at any of them. Hence, $L$ is the kind of pencil we have considered before (part 3D), viz., one in which each singular fibre has one ordinary double point off the base.

We will refer to such a pencil simply as general (with respect to $V$ ).

B. Now if we associate to a point in $V$ the point in $L$ on whose section it lies, we get a rational map $f: V \rightarrow L$ whose fibres are the hyperplane sections. We can pick a base-point in $L$, make cuts in $L$, look at the corresponding cones in $V$, etc.

More generally, we can do what was mentioned in 3B: let $H \in P_{N}^{*}$ (and suppose $V \notin H$ ). Then $V \cap H$ is locally a deformation retract in $V$ and if $U$ is any pre-assigned neighborhood of $V \cap H$, then $V \cap H$ will be $\subseteq U$ as soon as $H^{\prime}$ is near enough to $H$ in $P_{N}^{*}$.

This means that if $g:[0,1] \rightarrow P_{N}^{*}$ is a family of hyperplanes and if $g(u) r$ $V$ has the same kind of singularities as $g\left(u^{\prime}\right) \cap V$ for $u, u^{\prime} \in[0,1)$, then there are induced homeomorphisms fro a $g(u) \cap V$ to $g\left(u^{\prime}\right) \cap V$ and an induced map from $g(u) \cap V$ to $g(1) \cap V(u \in[0,1])$. We get the usual collection of vanishing cycles, cones, etc.

For example, if $g(u)$ is a nonsingular point in $V^{*}$ for all $u \in[0,1]$, then the function $b$ from $[0,1]$ to $V$ which assigns to each $u$ the double point of $g(u) \cap$ $V$ is continuous, and the rest of the homeomorphism between $g(u) \cap V$ and $g\left(u^{\prime}\right)$ $\cap V$ can be obtained by construction of the normal bundles in $V-b[0,1]$.

C. Suppose now that $V$ is no longer nonsingular and irreducible. What should $V^{*}$ mean? It cannot just mean those hyperplanes whose section on $V$ is 
singular, since if the dimension of the singular set of $V$ is $>0$, every hyperplane does that.

What $V^{*}$ ought to be is those sections which are "more singular" than the general section. I am not sure what that would be in general. Perhaps one could show that for all but a proper algebraic subset of $P_{N}^{*}$ the sections are all homeomorphic, or (if the Betti numbers are semicontinuous functions on $P_{N}^{*}$ ) have the same Euler characterist ic or middle homology group.

For instance, if $V$ has isolated singularities (or none), these criteria are all equivalent and $V^{*}$ is the planes tangent to $V$ at nonsingular points or passing through a singular point (i.e., cutting singular sections).

D. Furthermore, if one knew what $V^{*}$ was, one could then take a general pencil $L$ in which a finite number of sections would be "extra-singular." Again there would be vanishing cycles and cones with which one could hope to build the homology of $V$.

(3.9) would fail, but perhaps (3.10) could become

(4.1) $H_{i}(V, H \cap V)$ is generated by the cones for $i \leq \operatorname{dim} V$.

For example, suppose $V$ possesses an improper double point, i.e., one formed by taking a nonsingular $\bar{V}$ and identifying two of its points, $P$ and $Q$. An arc in $\bar{V}$ joining $P$ and $Q$ becomes a 1 -cycle in $V$ which cannot be pulled off the double point. So if $\operatorname{dim} V>1,(3.9)$ is violated, but clearly this 1 -cycle is homologous to a cone.

These ideas can be worked out for curves, at least, and also for certain simple kinds of singular varieties (see part 9).

5. The branch function.

A. We return to our pencil $V_{t}$ given by $f: W \rightarrow C$. For the present we allow a base locus but assume the general $V_{t}$ nonsingular (unless we can apply $4 \mathrm{C}$ and $\mathrm{D})$. We have the finite set $S$ whose fibres are singular. Then from $4 \mathrm{~A}$, $V_{t}^{*}(t \notin S)$ is a hypersurface in $P_{N}^{*}$ and forms an algebraic family (indexed by $C-S)$. Hence, all the $V_{t}(t \notin S)$ have the same class $m$, and when $t$ approaches a point 0 in $S, V_{t}^{*}$ becomes a well-defined (reducible) hypersurface in $P_{N}^{*}$ of degree $m$.

This hypersurface, of course, will be defined to be the dual of $V_{0}$ and denoted $V_{0}^{*}$. It is another candidate to be added to those suggested in 4C. How ever, it is not intrinsic, since it depends on the enveloping pencil $V_{t}$, at least if there is a base locus $B$ present. In general, $V_{0}^{*}=$ dual of $V_{0}$ (viz. 4C) + dual of singular locus of $B$ (viz. 4C). See part 9 below for a special case.

B. The duals $V_{t}^{*}$ now form a 1-parameter family of hypersurfaces of degree $m$ indexed by all of $C$. Let $L$ be a line in $P_{N}^{*}$ in general position with respect to this family. 
(5.1) Theorem. Every $V_{t}^{*}$ meets $L$ in a finite number of points (which, of course, is always $\leq m$ ).

(5.2) Proof. The Grassmann variety $G(1, N)$ of lines in $P_{N}$ is of dimension $2 N-2$. Those lines contained in some $V_{t}^{*}$ form an algebraic subset $\mathcal{L}$ of $G(1, N)$. We need only show that $\operatorname{dim} \varrho \leq 2 N-3$.

The principle of counting constants shows that we need only establish

(5.3) Lemma. Let $V$ be a bypersurface in $P_{N}$. Then the lines in $V$ form $\&$ an algebraic subset $\&$ of $G(1, N)$ of dimension $\leq 2 N-4$.

(5.4) Proof. Let $H$ be a hyperplane and $p$ a point not in $V$ or $H$. Then if $L$ is any line in $V, p$ and $L$ span a 2-plane which meets $H$ in a line. We thus establish a rational map $b$ from $£$ to $G(1, N-1)$ which is everywhere defined. Since $\operatorname{dim} G(1, N-1)=2 N-4$, we need only show that $b^{-1}(L)$ is finite, where $L$ is a line in $H$.

If not, then $b^{-1}(L)$ would contain a 1 -parameter family of lines, a ruled subsurface $R$ of $V$ which $p$ projects onto the line $L$. Then $R$ is a subset of the 2-plane $(p, L)$ spanned by $p$ and $R$, hence, $R=(p, L)$. But a (nontrivial) 1 parameter family of lines in a plane must cover it, i.e., one of the lines of $b^{-1}(L)$ passes through $p$. But this contradicts the assumption that $p \notin V$.

C. (5.1) shows that $\left\{V_{t}^{*} \cap L\right\}, t \in C$, is a $\gamma_{m}^{1}$ on $L$, i.e., a 1-parameter family of divisors of degree $m$, if $L$ is general. It clearly has no base points, since these would be common to all the $V_{t}^{*}$. But $\bigcap_{t \in C} V_{t}^{*}$ is of codimension $\geq 2$ in $P_{N}^{*}$, so a general line misses it.

All but a finite number of the $m$-tuples in $\gamma_{m}^{1}$ consist of $m$ distinct points, i.e., $L$ is general with respect to all but a finite number of the $V_{t}^{*}$.

Let $\bar{S}=$ those $t$ for which $L$ is not general. Clearly, $S \subseteq \bar{S}^{t}$. Let $u$ be a coordinate on $L$, so we can consider $u$ as a rational map from $W$ to $L$ whose fibres are the hyperplane sections. Then $\gamma_{m}^{1}$ defines an algebraic correspondence between $C$ and $L: t \in C$ and $u \in L$ are associated if $u \in V_{t}^{*}$. In this way $u$ becomes an algebraic function $u(t)$ on $C$ and $\bar{S}$ is where $u(t)$ ramifies.

(5.5) Definition. $u(t)$ is called the branch function of the pencil $V_{t}$ relative to $L$. The correspondence curve $J=\left\{(t, u) \in C \times L \mid u \in V_{t}^{*}\right\}$ is called the branch curve.

D.

(5.6) Theorem. The branch curve is irreducible.

(5.7) Proof. We extend the correspondence $J$ as follows:

Let $M$ be a general 2-plane through $L$ and consider the family $V_{t}^{*} \cap M$ in $M$. By (5.1), $L$ is not contained in any component of the $V_{t}$, so $M$ is not either. 
Hence, each $V_{t}^{*} \cap M$ is a curve. Since a general $V_{t}^{*}$ is irreducible and $M$ is general, a general member of $\left\{V_{t}^{*} \cap M\right\}$ is also irreducible.

As before, we have a correspondence between $C$ and $M$, viz., $\bar{J}=\{(t, v) \epsilon$ $\left.C \times M \mid v \in V_{t}^{*}\right\}$. Its projection onto $M$ is a finite covering, except that for finitely many $v \in M, C \times v \subseteq \bar{J} \cdot \bar{J} \cap(t \times M)=V_{t}^{*} \cap M$, so $\bar{J}$ is an irreducible surface (note that no $t \times M$ is a component of $\bar{J}$ ).

Hence, we obtain a 2-dimensional linear system of curves on $\bar{J}$ (or on its desingularization) by pulling back the lines in $M$. But then Bertini's theorem says that the general member, i.e., $J$, is irreducible.

6. Application of the branch function.

A. We now describe how the branch function can be used to examine the homology behavior of the $V_{t}$.

Let $t_{0} \notin \bar{S}$, so that $L$ is general for $V_{t_{0}}$. Mark the $m$ points $u\left(t_{0}\right)$ in $L$

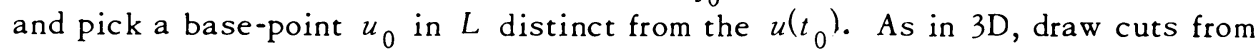
$u_{0}$ to the $u\left(t_{0}\right)$. If we number the $u\left(t_{0}\right)$ as $u_{1}\left(t_{0}\right), \cdots, u_{m}\left(t_{0}\right)$, let the corres ponding cuts be $g_{1}, \cdots, g_{m}$. Now as $t$ moves in $C-\bar{S}$, starting from $t_{0}$, the $u_{i}(t)$ will vary continuously and never coincide, so we can make $u_{0}$ and the $g_{i}$ vary continuously with $t$ also, such that at any stage the $g_{i}(t)$ form an appropriate set of cuts in $L$ centered at $u_{0}(t)$.

Let $d_{i} \in H_{n-2}\left(H_{u_{0}} \cap V_{t_{0}}\right)$ be the vanishing cycle along $g_{i}\left(t_{0}\right)$. Then, as in $3 \mathrm{~B}$, as $t$ moves in $C-\bar{S}$, the $d_{i}$ will vary continuously so that $d_{i}(t) \epsilon$ $H_{n-2}\left(H_{u_{0}(t)} \cap V_{t}\right)$ and $d_{i}(t)$ vanishes along $g_{i}(t)$. Then the corresponding cones $D_{i}(t)$ form a continuous family of $(n-1)$-chains, and if $\Sigma n_{i} D_{i}\left(t_{0}\right)$ is a cycle in $H_{u_{0}} \cap V_{t_{0}}$, then $\sum n_{i} D_{i}(t)$ forms a continuous family of $(n-1)$-cycles.

Suppose $t$ traverses a loop $g$ based at $t_{0}$. Then the points $u_{i}(t)$ describe $m$ arcs in $L$, so there are points of $L$ not on these arcs. This means that the base-point $u_{0}(t)$ can be chosen independent of $t$, i.e., is constantly equal to $u_{0}$ as $t$ moves on $g$. Furthermore, we can choose $u_{0}$ so that $H_{u_{0}} \cap W$ is nonsingular, since $L \notin W^{*}$. Hence, the $d_{i}(t)$ move in the pencil $H_{u_{0}}^{u_{0}} \cap V_{t}$ and we have the basis for an inductive procedure.

The Lefschetz theorems of $3 \mathrm{D}$ show that any $i-$ cycle of $V_{t}(i \leq n-2)$ can be put in $H_{u_{0}} \cap V_{t}$, so the behavior of $H_{i}\left(V_{t}\right)$ can be reduced to a pencil of lower dimension. As for $H_{i}\left(V_{t}\right)(i>n-1)$, the P-L transformation on it is adjoint to that on $H_{2 n-2-i}\left(V_{t}^{i}\right)$ (at least mod torsion), so is determined by what happens on the first half of the homology sequence.

B. Thus, our problem becomes that of investigating the change in the cones over the $g_{i}$, i.e., the group $H_{n-1}\left(V_{t}, H_{u_{0}} \cap V_{t}\right)$.

When $t$ traverses $g$, the branch points $u_{i}\left(t_{0}\right)$ undergo a permutation $\pi$ and 
the original cuts $g_{i}$ are transformed into a new system of cuts $g_{\pi(i)}^{\prime}$. The original vanishing cycles $d_{i}$ and cones $D_{i}$ become $d_{\pi(i)}^{\prime}$ and $D_{\pi(i)}^{\prime}$. We must express the $D_{i}^{\prime}$ in terms of the original $D_{i}$.

This is done as follows (cf. (3.11)):

Consider an arc $g^{\prime}$ in $L$ from $u_{0}$ to $u_{1}\left(t_{0}\right)$, say. A certain $(n-2)$-cycle $d$ vanishes along $g^{\prime}$. Now in $L-\bigcup_{i>1} u_{i}\left(t_{0}\right), g^{\prime}$ is homotopic to $b \circ g_{1}$ where $b$ is a loop at $u_{0}$.

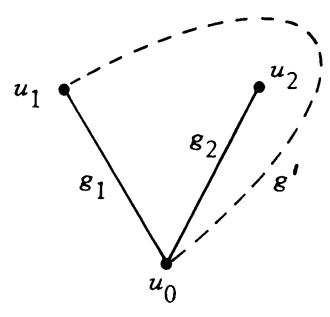

Figure 1

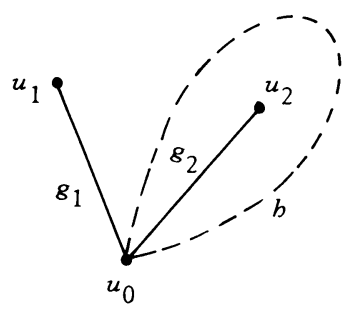

Now after $t$ traverses $h, d$ becomes a cycle $\bar{d}$ which vanishes along $g_{1}$. Hence, $\bar{d}= \pm d_{1}$. Choose $d$ so that $\bar{d}=d_{1}$. Then $D\left(d, g^{\prime}\right)=D_{1}+D(d, b)$. So we must reduce $D(d, b)$ to a sum of the $D_{i}$.

$b$, being an element of the fundamental group $\pi_{1}\left(L-u\left(t_{0}\right), u_{0}\right)$, is generated by loops $l_{i}$, where $l_{i}$ encloses just $g_{i}$. Hence, $D(d, b)=\Sigma D\left(\bar{d}_{i}, l_{i}\right)$, but as in 3D, $D\left(\bar{d}_{i}, l_{i}\right)= \pm\left(\bar{d}_{1}, d_{i}\right) D_{i}$ and we are done.

C. Let us turn to the case at hand, where $g$ is a small loop about a point 0 of $S$.

By the classical theory of algebraic functions of one variable, the branch function $u(t)$ can be expressed (near 0 ) by a collection of Puiseux series or cycles. We can choose the coordinate $u$ on $L$ so that $H_{\infty}$ is not tangent to any $V_{t}, t$ small. This means that the Puiseux series have no negative terms, i.e., have finite values at $t=0$. These values, of course, are just the points $V_{0}^{*} \cap L$.

Consider one of these Puiseux series:

$$
u(t)=c_{1} t^{a / n}+c_{2} t^{a} 2^{/ n}+\cdots,
$$

where the $c_{i}$ are nonzero constants and the $a_{i}$ are integers with $a_{1}<a_{2}<\cdots$. Obviously, we can take the integers $n, a_{1}, a_{2}, \ldots$ relatively prime (so this cycle effectively contains $n$ points of $V_{t}^{*} \cap L$ ). Furthermore, by choosing the $u$ coordinate so that $u(0)=0$, we can assume $a_{1}>0$.

These $n$ points behave rather simply as $t$ traverses $g$, viz., they undergo a cyclic permutation. But it is more difficult to say what happens to the cuts $g_{i}$. (See 7A for discussion and examples.) Here we will only discuss the case that we will need later (part 9). 
D. The simplest behavior occurs when $a_{1}=1$. This just means that $(0,0)$ is a simple point on $J$. We can change the coordinate $t$ on $C$ so that (6.1) becomes $u=t^{1 / n}$. The branch points then move in circles (if $t$ does) and it is easy to choose cuts which behave nicely.

Suppose the points are numbered 1 through $q$ and undergo the permutation $(12 \cdots q)$. For convenience, we picture them in a row (see Figure 2),

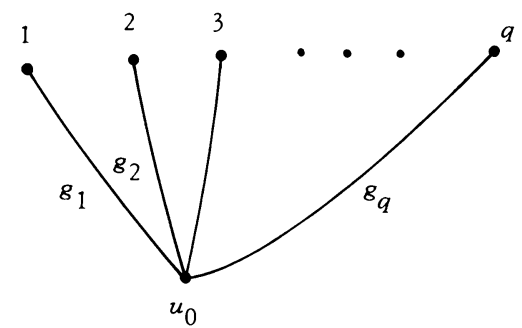

Figure 2

and draw the cuts $g_{i}$ so that $g_{1} \rightarrow g_{2} \rightarrow \cdots \rightarrow g_{q}$ as $t$ moves about 0 . Furthermore, if $d_{1}$ vanishes along $g_{1}$ and $d_{2}, d_{3}, \ldots \cdot$ are its transforms in $H_{n-2}\left(V_{t} \cap H_{u 0}\right)$ as $t$ circles 0 , then $d_{i}$ vanishes a long $g_{i}$, so the corresponding cones also undergo the transformation $D_{1} \rightarrow D_{2} \rightarrow \cdots \rightarrow D_{q}$.

The key question, then, is: what happens to $D_{q}$ ? The answer is $D_{q} \rightarrow D_{q+1}$ $=D\left(d_{q+1}, g_{q+1}\right)$, where $d_{q+1}$ and $g_{q+1}$ are the $q$ th transforms of $d_{1}$ and $g_{1}$, respectively.

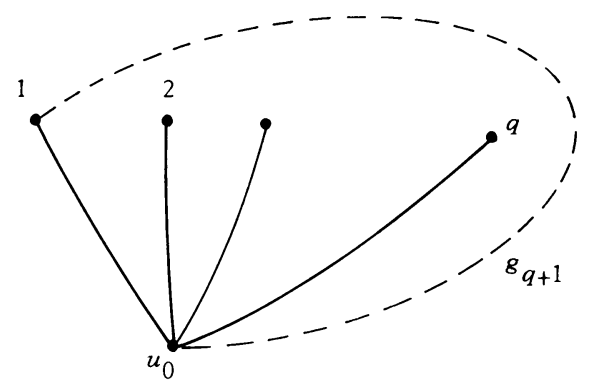

Figure ?

Hence, $D_{q+1}= \pm D_{1}+b_{2} D_{2}+\cdots+b_{q} D_{q}$ for some integers $b_{i}$.

$E$. The precise determination of the $b$ 's depends on knowing the intersection numbers of the $d_{i}$. But we can still draw some conclusions about the P-L transformation without knowing them.

Clearly $D_{q+1}=D\left(d_{q+1}, b\right) \pm D_{1}$, where $b$ is a loop in $L$ enclosing all the branch points of this cycle. When $t$ stays close to 0 , the branch points and their cuts will stay inside this loop. Hence, $b$ is an invariant path with respect to the change in $t$, so if $T$ is the $\mathrm{P}-\mathrm{L}$ transformation, we have

(6.2) Proposition. (1) $\left(T^{q} \pm 1\right) D_{1}=D\left(T^{q+1} d_{1}, b\right)$,

(2) $T\left(D\left(T^{q} d_{1}, b\right)\right)=D\left(T^{q+1} d_{1}, b\right)$. 
Thus, if $P(T)$ is the minimal polynomial for $T$ on the homology of $H_{u_{0}} \cap V_{t}$ (or just on $d_{1}$ ), we have

$$
P(T)\left(T^{q} \pm 1\right) D_{1}=0 .
$$

We conclude that if Theorems I and II of the introduction are true in dimension $<\operatorname{dim} W$, they are true for the pencil $\dot{V}_{t}$ if the branch curve is nonsingular.

7. Examples of branch curves.

A. In general, we cannot assume that the branch curve is nonsingular or that the branch points move in circles. Even worse, although $J$ is irreducible, it may not be so locally and several of the Puiseux series (6.1) may have the same center. So the cuts drawn to one cycle may interfere with those drawn to another of the same center. We give some examples of this behavior.

B. Consider the pencil of cubics

$$
t x^{3}+y^{3}+z^{3}=0
$$

in the projective plane $(=W)$. When $t=0$, the fibre $V_{0}$ is $y^{3}+z^{3}=0$, or three lines concurrent at $p=(1,0,0)$.

The general hyperplane pencil $H_{u}$ is just the set of lines in $W$ through some point. Choose the parameter $u$ so that $H_{0}$ goes through $p$.

(7.2) Proposition. $V_{0}^{*}=6 L_{0}$, where $L_{0}$ is the line in $W^{*}$ representing the lines in $W$ through $p$.

(7.3) Proof. We calculate the dual of the general $V_{t}$. The generic point of $V_{t}^{*}$ is $\left(t x^{2}, y^{2}, z^{2}\right)$, where $t x^{3}+y^{3}+z^{3}=0$. If $(A, B, C)$ are the coordinates in $W^{*}$, then $V_{t}^{*}$ is given by $(t A)^{3 / 2}+B^{3 / 2}+C^{3 / 2}=0$, which is equivalent to the polynomial relation

$$
A^{6}-2 t A^{3}\left(B^{3}+C^{3}\right)+t^{2}\left(B^{3}-C^{3}\right)^{2}=0 .
$$

When $t=0$, we get the curve $A=0$ counted 6 times. But $A=0$ is just the line $L_{0}$ in $W^{*}$.

In order to find the Puiseux expansions around $t=0$, we write the line $H_{u}$ parametrically as

$$
A=u, B=a u+b, \quad C=c u+d
$$

and substitute in (7.1). The resulting expression in $t$ and $u$ is the local equation for $J$ at $(0,0)$.

One easily sees that

(7.6) $(0,0)$ is a double point on $J$ with double points in the first two neighborhoods. $u$ is given by two Puiseux series $u_{i}(t)=e_{i} t^{1 / 3}+\cdots\left(e_{1} \neq e_{2}\right)$. 
Here, the six branch points travel in noninterfering circles about 0 , but undergo the permutation (135)(246) instead of (123456).

This kind of behavior allows the cuts to be generated by two of their number, i.e., we draw $g_{1}$ and $g_{2}$ as in part 6D (Figure 2) and observe that $g_{1}$ becomes $g_{3}, g_{5}, \ldots$ and $g_{2}$ becomes $g_{4}, g_{6}, \ldots$ as $t$ moves about 0 . Hence, the reasoning and conclusions of $6 \mathrm{E}$ are still valid.

Notice that all this goes through for any $V_{0}$ with an ordinary triple point off the base, since the question is local.

C. More generally, suppose $V_{0}$ is an $s$-fold curve (or component) with an ordinary $r$-fold point $p$.

Let $x$ and $y$ be affine coordinates in $W$ at $p$ and take the pencil $V_{t}$ to be

$$
\left(x^{r}+y^{r}\right)^{s}=t
$$

This special form will not affect the properties of the branch function, but makes calculation easier.

(7.8) Proposition. For each $V_{t}(t \neq 0)$ there are $r s(r-1)$ branch points near $p$ distributed in $r-1$ Puiseux series each varying like $t^{1 / r s}$. The motion of the branch points and cuts is like the $(r-1)$ st power at a simple branch point of order $r s(r-1)$, so the conclusions of $6 \mathrm{E}$ bold.

(7.9) Proof. Let the lines $H_{u}$ be given by $x=u, y=a u+b$.

The branch curve is the locus where $V_{t}$ and $H_{u}$ are tangent, i.e.,

$$
\operatorname{rank} \frac{\partial\left(V_{t}, H_{u}\right)}{\partial(x, y)}<2 \text {. }
$$

The Jacobian here is

$$
\left(\begin{array}{cc}
r s\left(x^{r}+y^{r}\right)^{s-1} x^{r-1} & r s\left(x^{r}+y^{r}\right)^{s-1} y^{r-1} \\
a & -1
\end{array}\right)
$$

Hence, $x^{r-1}+a y^{r-1}=0$, since $x^{r}+y^{r} \neq 0$ (we are not on $V_{0}$ ). The branch curve then consists of $r-1$ lines through $p$. Let $y=c x$ be one of them. Substituting in (7.7) gives $x^{r s}=c^{\prime} t$ for some constant $c^{\prime} \neq 0$, or $u=c^{\prime \prime} t^{1 / r s}$. This constant $c^{\prime \prime}$ is obviously different for each of the $r-1$ lines.

D. However, the branch points do not even have to move in a single circle. For example, take the case of plane quartics splitting into a cuspidal cubic plus a line through the cusp (or the corresponding local situation).

If the line is not the cuspidal tangent, we can take $V_{t}$ to be

$$
\left(y^{2}-x^{3}\right) x=t
$$


(7.11) Proposition. There are two Puiseux series centered at the cusp. One is $t^{1 / 3}$ and one is $t^{1 / 4}$.

(7.12) Proof. Take the hyperplane pencil as in (7.9).

The Jacobian is

$$
\left(\begin{array}{cc}
y^{2}-4 x^{3} & 2 x y \\
a & 1
\end{array}\right)
$$

so the branch curve is

$$
y^{2}-4 x^{3}-2 a x y=0
$$

It has an ordinary double point at the origin with tangents $y=0$ and $y=2 a x$. The branch points on the $y=0$ branch move like $t^{1 / 3}$ and those on $y=2 a x$ like $t^{1 / 4}$.

E. If the line through the cusp is the cuspidal tangent, our pencil becomes

$$
\left(y^{2}-x^{3}\right) y=t
$$

and the Jacobian is

$$
\left(\begin{array}{cc}
-3 x^{2} y & 3 y^{2}-x^{3} \\
a & 1
\end{array}\right)
$$

The branch curve is $3 x^{2} y+3 a y^{2}-a x^{3}=0$. At the origin, this is parametrically $x=3 s^{2}+\cdots, y=s^{3}+\cdots$, and when substituted in (7.13) becomes

$$
-26 s^{9}+\cdots=t \text { or } s=\text { P.S. }\left(t^{1 / 9}\right) \text {. }
$$

Then $x=c_{1} t^{2 / 9}+\cdots, y=c_{2} t^{3 / 9}+\cdots$ and $u=a c_{1} t^{2 / 9}+\cdots$ Here, the branch curve is locally irreducible.

\section{Ordinary double points.}

A. We apply the method of construction of homology to examine the case where $V_{0}$ has an ordinary double point off the base and demonstrate the results of $3 \mathrm{C}$.

Choose the general hyperplane pencil $H_{u}, u \in L$. We can assume that no member of $L$ is tangent to $V_{0}$ at points in the base of $V_{t}$ or the base of $L$ or at points where $L$ is tangent to $W$.

(8.1) Proposition. If $x$ is a simple point of $V_{0}$ at which some $H_{u}$ is tangent, then the Puiseux series $u(t)$ at $x$ is holomorphic.

(8.2) Proof. Let $z_{1}=0$ be the equation of $V_{0}$ at $x$, so the $V_{t}$ is $z_{1}=t$. If $H_{0}$ is tangent to $V_{0}$ at $x$, then $H_{u}$ is given by $u=z_{1}+$ h.p. Substituting $t$ 
for $z_{1}$ gives $u=b(t)+G$, where $b$ is a power series in $t$ and $G$ is a power series in $t, z_{2}, \cdots, z_{n}$ of degree $>1$ in the $z^{\prime}$ s.

This is the local equation (in $V_{t}$ ) of $V_{t} \cap H_{u}$. Hence, it has a singular point near $x$ if and only if $u=b(t)$, so $u$ is indeed holomorphic in $t$.

(8.3) Proposition. The cone over the path from $u_{0}$ to $u(t)$ is invariant.

(8.4) Proof. (8.1) says that the path is invariant. On the other hand the vanishing cycle over the path is invariant, since $V_{0} \cap H_{u_{0}}$ is nonsingular in the pencil $V_{t} \cap H_{u_{0}}$.

B. The only point left to consider is the double point $p$ itself.

(8.5) Proposition. The brancb curve is nonsingular at $p$ and $u$ is a power series in $t^{1 / 2}$.

(8.6) Proof. By 4A, we can take the pencil to be $z_{1}^{2}+z_{2}^{2}+\cdots+z_{n}^{2}=t$.

Let $H_{u}$ be $u=\Sigma c_{i} z_{i}+$ h.p., so that $H_{0}$ goes through $p$. The hyperplane pencil being general, we have that $H_{0}$ is not tangent to the tangent cone at $p$ in $V_{0}$, i.e., $\Sigma c_{i}^{2} \neq 0$.

The Jacobian is

$$
\left(\begin{array}{ccc}
2 z_{1} & \cdots & 2 z_{n} \\
c_{1}+\cdots & & c_{n}+\cdots
\end{array}\right) .
$$

When its rank is 1 , we have

$$
z_{i}=\lambda\left(c_{i}+\cdots\right)
$$

for some $\lambda$ and all $i$. We are only interested in small $\lambda$, since the branch points we want are close to $p$.

The coefficient matrix of the linear part of (8.7) is of the form $I-\lambda M$, where $I$ is the identity $n \times n$ matrix and. $M$ is the Hessian of $H_{0}$ at $p$. Hence, $\operatorname{det}(I-\lambda M) \neq 0$ for small $\lambda$, which means that (8.7) can be uniquely inverted near $p$ to yield the branch curve whose parametric equations are $z_{i}=c_{i} \lambda+\cdots$, all $i$. Hence, $t=\Sigma c_{i}^{2} \lambda^{2}+$ h.p. and $u=\Sigma c_{i}^{2} \lambda+$ h.p. and $u$ is a power series in $t^{1 / 2}$.

Note that we could have used the simpler pencil $u=z_{2}$. The branch function is then just $u=t^{1 / 2}$. This device of using simpler local equations will be helpful later on.

C. Draw the cuts $g_{1}$ and $g_{2}$ as in $6 \mathrm{D}$ and then any others needed for the other branch points.

If an element $D$ of $H_{n-1}\left(V_{t}, V_{t} \cap H_{u_{0}}\right)$ is written in the form $D=q_{1} D_{1}+$ $q_{2} D_{2}+\cdots$, then by $(8.3),(T-1) D=q_{1}(T-1) D_{1}+q_{2}(T-1) D_{2}$. We have $T\left(D_{1}\right)=D_{2}$ (by 6D). What is $T\left(D_{2}\right)$ ? 
There are two cases, depending on whether $n$ is even or odd.

(8.8) Proposition. If $n$ is odd, $T\left(D_{2}\right)=2 D_{2}-D_{1}$. If $n$ is even, $T\left(D_{2}\right)=$ $D_{1}$.

(8.9) Proof. We use induction. Let $d$ vanish along $g_{1}$ and generate $D_{1}$. Then $d$ also generates $D_{2}$. If $n$ is odd, then (3.7) says that $d$ goes to $-d$ after $u$ goes around $g_{2}$, while if $n$ is even $d$ is invariant around $g_{2}$. In either case (8.8) follows.

The discussion of part $4 \mathrm{D}$ applies to $V_{0}$, as can be seen by blowing up its singular point and noting that the pencil $H_{u} \cap \bar{V}_{0}$ is still sufficiently general in the sense of Lefschetz (see (3.8)). The section $H_{0} \cap V_{0}$ has an ordinary double point, so as $H_{u} \cap V_{0}$ approaches $H_{0} \cap V_{0}$, a single cycle will vanish and trace out a cone $D_{0}$ on $V_{0}$. The path of approach from $u_{0}$ to 0 (in $L$ ) can be taken to be $g_{0}$, near $g_{1}$ and $g_{2}$.

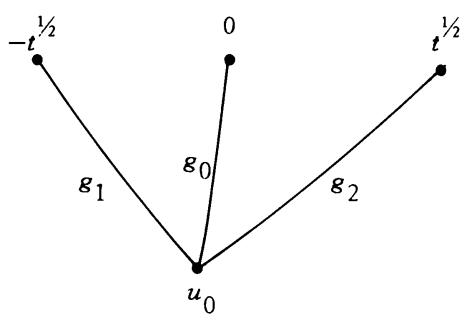

Figure 4

Hence, as $t \rightarrow 0, d$ will approach $d_{0}$ and $D_{1}$ and $D_{2}$ will become $D_{0}$. In other words, $D_{1}-D_{2}$ is an $(n-1)$-cycle on $V_{t}$ which vanishes at $V_{0}$. Since $D_{1}$ and $D_{2}$ are cones over a sphere, $D_{1}-D_{2}$ is topologically a sphere, too. (8.8) then yields

(8.10) If $n$ is odd, $T\left(D_{1}-D_{2}\right)=-\left(D_{1}-D_{2}\right)$. If $n$ is even, $T\left(D_{1}-D_{2}\right)=$ $D_{1}-D_{2}$.

This verifies most of $3 \mathrm{C}$ except for the intersection numbers.

D.

(8.11) Proposition. $D_{1}-D_{2}$ can be pulled off any algebraic subset $A$ of $V_{t}$

(8.12) Proof. Move the base-point $u_{0}$ slightly. Then $d, D_{1}$ and $D_{2}$ will vary continuously into $\bar{d}, \bar{D}_{1}$ and $\bar{D}_{2}$.

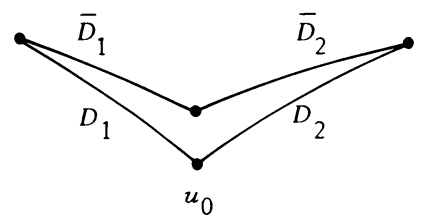

Figure 5 
Hence, $D_{1}-D_{2}=\bar{D}_{1}-\bar{D}_{2}$ in $H_{n-1}\left(V_{t}\right)$. Since by induction $d$ can be pulled off $V_{t} \cap H_{u} \cap A$ for each $u$ (except perhaps the singular points), we need only choose the pencil $L$ so that its tangencies on $V_{t}$ miss $A$. Hence, all of $\bar{D}_{1}-$ $\bar{D}_{2}$ is disjoint from $A$.

This also shows that $D_{i} \cap\left(\bar{D}_{1}-\bar{D}_{2}\right)=\varnothing$ if $i>2$, so we need only check (3.6) for $D_{1}$ and $D_{2}$.

We have $T\left(D_{1}\right)=D_{1}-\left(D_{1}-D_{2}\right)$. Hence, we must show that $\left(D_{1}, D_{1}-D_{2}\right)$ $=(-1)^{(n-1)(n-2) / 2}$. $\left.\bar{D}_{1}\right)$.

This intersection number is the same as $\left(D_{1}, \bar{D}_{1}-\bar{D}_{2}\right)$ which is also $\left(D_{1}\right.$,

(8.13) $\left(D_{1}, \bar{D}_{1}\right)=(-1)^{(n-1)(n-2) / 2}$.

(8.14) Proof. Choose coordinates $z_{1}, \cdots, z_{n}$ at the vertex of $D_{1}$ so that $H_{u} \cap V_{t}$ is given in $V_{t}$ by $z_{1}^{2}+\cdots+z_{n-1}^{2}=u$, and the cone $\left\{z_{i}\right.$ real $\mid \Sigma z_{i}^{2}$ $\leq 1\}$ is the tip of $D_{1}$.

$\bar{D}_{1}$ is obtained by moving $u$ slightly counterclockwise from 1 and is given by

$$
\left\{\left(z_{1}, \cdots, z_{n-1}\right) \mid z_{1} u^{-1 / 2} \text { real and } \sum\left(z_{i} u^{-1 / 2}\right)^{2} \leq 1\right\}
$$

Hence, $\bar{D}_{1}=u^{1 / 2} D_{1}$.

Using Lefschetz's prescription for calculating intersection numbers [4], let $\left(P_{0}, \ldots, P_{n-1}\right)$ be an orientation for the cell $D_{1}$ where $P_{0}=(0, \cdots, 0)$. Then the orientation for $\bar{D}_{1}$ is $\left(P_{0}, u^{1 / 2} P_{1}, \cdots, u^{1 / 2} P_{n-1}\right)$.

Putting these together gives the orientation $\left(P_{0}, P_{1}, \ldots, P_{n-1}, u^{1 / 2} P_{1}\right.$, $\left.\cdots, u^{1 / 2} P_{n-1}\right)$ for $V_{t}$ near $P_{0}$. But the orientation $V_{t}$ has as a complex manifold is $\left(P_{0}, P_{1}, i P_{1}, \ldots, P_{n-1}, i P_{n-1}\right)=\left(P_{0}, P_{1}, u^{1 / 2} P_{1}, \ldots, P_{n-1}\right.$, $\left.u^{1 / 2} P_{n-1}\right)$, since $u^{1 / 2}$ is counterclockwise from 1 . To change the first orientation to the second requires $(n-2)+(n-3)+\cdots+2+1+0$ transpositions, so $\left(D_{1}, \bar{D}_{1}\right)=(-1)^{(n-1)(n-2) / 2}$.

Similarly, (3.6) can be checked for $D_{2}$, since $\left(\bar{D}_{2}, D_{2}\right)=(-1)^{(n-1)(n-2) / 2}$.

(8.15) Corollary. (3.7) bolds.

(8.16) Proof. When $n$ is even, this is clear, since $D_{1}-D_{2}$ is then odddimensional and so always has self-intersection number 0 .

When $n$ is odd, $\left(D_{1}-D_{2}, D_{1}-D_{2}\right)=\left(D_{1}-D_{2}, \bar{D}_{1}-\bar{D}_{2}\right)=\left(D_{1}, \bar{D}_{1}\right)+\left(D_{2}\right.$, $\left.\bar{D}_{2}\right)=2(-1)^{(n-1)(n-2) / 2}$. But $(n-1)(n-2) / 2 \equiv(n-1) / 2(\bmod 2)$, when $n$ is odd.

9. Normal crossings.

A. Suppose there is no base in the pencil $V_{t}$, so $f$ is everywhere defined. The fibre $V_{0}$ can have arbitrary singularities, but we can use Hironaka's results 
on the resolution of singularities [2] which say that by repeated monoidal transformations centered in $V_{0}$, we can transform $W$ to a nonsingular $\bar{W}$ with a birational morphism $g: \bar{W} \rightarrow W$ such that

(9.1) (1) $g$ is an isomorphism between $\bar{W}-g^{-1}\left(V_{0}\right)$ and $W-V_{0}$.

(2) $g^{-1}\left(V_{0}\right)$ is a divisor on $\bar{W}$ with only normal crossings as singularities (see below for definition).

Now $b=f \circ g$ is a morphism from $\bar{W}$ to $C$ and so induces a pencil of fibres $\bar{V}_{t}$ in $\bar{W}$. By (9.1), $\bar{V}_{0}=g^{-1}\left(V_{0}\right)$ and $\bar{V}_{t} \simeq V_{t}, t \neq 0$. W and $\bar{W}$ are the same fibre space over $C-\{0\}$, so the P-L transformation on $V_{t}$ is the same as that on $\bar{V}_{t}$.

Hence, we can assume from the start that $V_{0}$ bas only normal crossings.

B.

(9.2) Definition. The divisor $V_{0}$ is said to consist of normal crossings if its support is the union of nonsingular hypersurfaces of $W$ which are transversal at every point.

This means that if $V^{1}, \cdots, V^{s}$ are the components of $V_{0}$ containing $x \in$ $V_{0}$ and if $z_{i}=0(i=1, \cdots, s)$ is a local equation for $V^{i}$ at $x$, then the $z_{i}$ are a partial system of coordinates at $x$ in $W$.

Suppose that all the components of $V_{0}$ are $V^{1}, \ldots, V^{r}$ and that $V^{i}$ has multiplicity $m_{i}>0$. Then near $x$, the pencil $V_{t}$ can be given by $z_{1}^{m_{1}} \cdots z_{s}^{m_{s}}$ $=t . \quad x$ is then $\left(m_{1}+\cdots+m_{s}\right)$-fold singular for the divisor $V_{0}$ and $s$-fold singular for its support $\left|V_{0}\right|$. Conversely, a point which is $s$-fold on $\left|V_{0}\right|$ belongs to exactly $s$ components of $V_{0}$.

Let $M_{i}=$ set of points $i$-fold singular for $\left|V_{0}\right|(i=1, \cdots, n)$. The $M_{i}$ possess the following properties:

(9.3) (1) The y are disjoint.

(2) $\bigcup_{i} M_{i}=\left|V_{0}\right|$.

(3) If $M_{i} \neq \varnothing$, it is a nonsingular locally closed algebraic subset of $W$ of dimension $n-i$.

(4) $\partial M_{i}=M_{i+1} \cup \cdots \cup M_{n}$.

(5) Each component of $\bar{M}_{i}^{n}$ is nonsingular.

C. Thus, we have a natural partition of $V_{0}$ into its singular subvarieties. It is clear what $V_{0}^{*}$ should be, viz., those hyperplanes tangent to some component of some $\bar{M}_{i}$. We shall show this later.

We first need some information about ge neral hyperplane pencils.

(9.4) Proposition. Let $V_{1}, V_{2}, \ldots$ be a finite number of nonsingular irreducible subvarieties of $P_{N}$ and $A_{i} \subseteq V_{i}$ proper algebraic subsets. Then in a general pencil of byperplanes, the points of tangency on $V_{i}$ do not lie on $A_{i}$. 
(9.5) Proof. Each $V_{i}^{*}$ is an irreducible subvariety in $P_{N}^{*}$ and the hyperplanes tangent to $V_{i}$ at a point of $A_{i}$ form a proper algebraic subset $A_{i}^{*}$ of $V_{i}^{*}$. Hence, the general line in $P_{N}^{*}$ misses every $A_{i}^{*}$.

We apply this to the collection of components of the $\bar{M}_{i}$. Let the $A$ 's be the parts of these components in $\partial M_{i}$. (9.4) then yields

(9.6) Corollary. Let $H_{u}$ be a general pencil of byperplanes. Then if $H_{u}$ is tangent to a component of $\bar{M}_{i}^{u}$, the point of tangency lies in $M_{i}$.

Furthermore, those hyperplanes tangent to more than one of these components form a subset of $P_{N}^{*}$ of dimension $<N-1$, so the pencil $L$ misses it. Hence,

(9.7) Each $H_{u}$ is tangent to at most one component of the $\bar{M}_{i}$ and the tangent section will have a single ordinary double point not lying on the base locus $B(L)$ of the pencil $H_{u}$. In particular, no point of $M_{n}$ is in $B(L)$.

D. We now examine the branch curve for $V_{t}$.

(9.8) Proposition. Let $x \in V_{0} \cap B(L)$. Then $x$ is not on the branch curve.

(9.9) Proof. Let $x$ belong to $V^{1}, \ldots, V^{s}$, so $V_{t}$ is $z_{1}^{m_{1}} \cdots z_{s}^{m_{s}}=t$ near $x$. Now $s<n$, or else $x \in M_{n} \cap B(L) . M_{s}$ is given locally by $z_{1}=\cdots=z_{s}=$ 0 . If $b=0$ is a local equation for one of the hyperplanes $H$, then since $H \cap M_{s}$ has no singularity at $x$, the function $z_{1}, \cdots, z_{s}, b$ form a partial system of coordinates there. Let us rename $b$ and call it $z_{s+1}$. Complete to a full set of coordinates $z_{1}, \cdots, z_{n}$.

Let $b^{\prime}=c_{i} z_{i}+$ h.p. $=0$ be the local equation for another hyperplane in $L$. For some $i \neq s+1, c_{i} \neq 0$. Otherwise, $b^{\prime}-c_{s+1} z_{s+1}=0$ is a hyperplane in $L$ tangent to $W$ at $x \in B(L)$, which contradicts the generality of $L$. Also, $i>s$, else $b^{\prime}=0$ would be tangent to $M_{s}$ at $x$.

$H \cap V_{t}$ is given by the same equation $z_{1}^{m_{1}} \cdots z_{s}^{m_{s}}=t$ in $V_{t}$, so $H$ is not tangent to $V_{t}, t \neq 0$. Every other hyperplane can be written in the form $b^{\prime}+$ $u z_{s+1}$. Hence, the Jacobian is

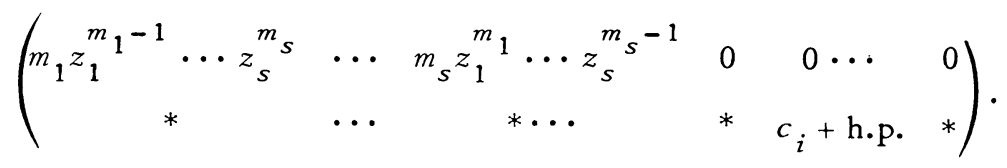

When $t \neq 0$, none of $z_{1}, \cdots, z_{s}$ is zero, while near $x, c_{i}+$ h.p. will be nonzero. Hence, this Jacobian has rank 2 and $x$ cannot be on the branch curve.

(9.10) Proposition. Suppose $x \notin B(L)$ but no $H_{u}$ is tangent to $M_{s}$ at $x$. Then $x$ is not on the branch curve. 
(9.11) Proof. The proof is the same as (9.9) except that now the hyperplane pencil takes the simpler form $u=z_{s+1}$. The Jacobian is

so is of rank 2.

$$
\left(\begin{array}{ccccccc}
m_{1} t / z_{1} & \cdots & m_{s} t / z_{s} & 0 & 0 & \cdots & 0 \\
0 & \cdots & 0 & 1 & 0 & \cdots & 0
\end{array}\right)
$$

E.

(9.12) Proposition. Suppose $x \notin B(L), x \in M_{s}$ and some byperplane in $L$ is tangent to $M_{s}$ at $x$. Then,

(1) $x$ belongs to the branch curve.

(2) If $V_{t}$ is $z_{1}^{m_{1}} \cdots z_{s}^{m_{s}}=t$ and $m=m_{1}+\cdots+m_{s}$, then $u$ is a Puiseux series in $t^{1 / m}$.

(3) $x$ is nonsingular on the branch curve and $u$ is a uniformizing parameter there.

(9.13) Proof. Let $H_{u}$ be $u=\Sigma c_{i} z_{i}+$ h.p. near $x$. If $H_{0}$ is tangent to $M_{s}$ at $x$, then $c_{s+1}=\cdots=c_{n}=0$.

If some $c_{i}(i \leq s)$ were zero, then $H_{0}$ would be tangent to some $M_{s^{\prime}}\left(s^{\prime}<s\right)$ at $x$. This contradicts (9.6). So none of $c_{1}, \cdots, c_{s}$ is zero.

The Jacobian is

$$
\left(\begin{array}{ccccccc}
m_{1} z_{1}^{m_{1}-1} \ldots z_{s}^{m_{s}} & \cdots & m_{s} z_{1}^{m_{1}} \cdots z_{s}^{m_{s}-1} & 0 & 0 & \cdots & 0 \\
c_{1}+\text { h.p. } & \cdots & c_{s}+\text { h.p. } & \frac{\partial u}{\partial z_{s+1}} & \cdots & \frac{\partial u}{\partial z_{n}}
\end{array}\right) .
$$

When $t \neq 0$, neither are $z_{1}, \cdots, z_{s}$, so the Jacobian has the same rank as

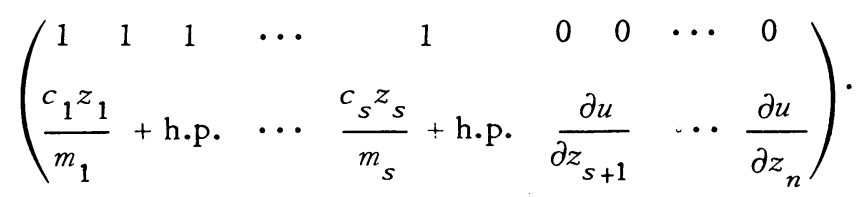

Hence, the points on the branch curve near $x$ are those satisfying the simultaneous equations

$$
\text { (9.14) } \lambda=c_{i} z_{i} / m_{i}+\text { h.p. }(i=1, \cdots, s) \text { and } \partial u / \partial z_{j}=0(j=s+1, \cdots, n)
$$
for some (small) $\lambda$.

$x$ satisfies the equations when $\lambda=0$, which establishes (1). For (2) and (3), we must solve for the $z$ 's in terms of $\lambda$.

The Jacobian of $(9.14)$ at $x$ is

$$
\left(\begin{array}{cccc}
c_{1} / m_{1} & \cdots & 0 & \\
0 & \cdots & c_{s} / m_{s} & \\
& 0 & & K
\end{array}\right),
$$


where $K$ is the Hessian of $u$ with respect to $z_{s+1}, \cdots, z_{n}$ evaluated at $x$. Hence, its determinant is

$$
\frac{c_{1} \cdots c_{s}}{m_{1} \cdots m_{s}} \operatorname{det} K \text {. }
$$

We already know that no $c_{i}=0$. But $K$ is also the Hessian of $H_{0} \cap M_{s}$ at $x$ in $M_{s}$, and since $x$ is an ordinary double point for $H_{0} \cap M_{s}$, its determinant is nonzero.

It follows that (9.14) can be inverted near $x$ to yield

(9.15) $z_{i}=d_{i} \lambda+$ h.p. $\left(d_{i}=m_{i} / c_{i}, i=1, \cdots, s\right)$ which are parametric equations for the branch curve. Substituting (9.15) in the expressions for $u$ and $t$, we get $u=m \lambda+$ h.p. and $t=c \lambda^{m}+$ h.p., $c \neq 0$. This gives (2) and (3).

(9.16) Note 1. We could have simplified the calculations had we taken $H_{u}$ to be, say, $z_{1}+\cdots+z_{s}+z_{s+1}^{2}+\cdots+z_{n}^{2}=u$, since this variety is also tangent to no $M_{s},\left(s^{\prime}<s\right)$ and cuts an ordinary double point on $M_{s}$.

Note 2. Since all the calculations are local, (9.12) also holds for those $V_{0}$ which consist of local normal crossings, i.e., such that locally $V_{0}$ is $z_{1}^{m_{1}} \ldots$ $z_{s}^{m_{s}}=0$ for some local coordinates $z_{i}$.

$\mathrm{F}$. The considerations of part $6 \mathrm{E}$ apply to the case of normal crossings since the branch curve is nonsingular at $t=0$ and the general section $H_{u_{0}} \cap V_{0}$ also has normal crossings. We know Theorems I and II ((1.6) and (1.8)) hold when $\operatorname{dim} W=1$ (part 2A).

Hence,

(9.17) Theorems I and II are established for arbitrary dimension.

(9.17) is equivalent to

(9.18) There is an integer $N$ such that the P-L transformation satisfies the relation $\left(T^{N}-1\right)^{n}=0, n=\operatorname{dim} W$.

10. The main theorems.

A. In order to prove Theorems I' and II' (part 1C) we must make a closer analysis of the behavior of the cones.

As in the previous part, consider the $m$ cones corresponding to a point $x$ on $M_{s}$ and $V_{t}: z_{1}^{m_{1}} \cdots z_{s}^{m_{s}}=t$. With respect to $L$ we have $m$ cuts $g_{i}$, vanishing cycles $d_{i}$ and cones $D_{i}$. If $T$ is the P-L transformation and $T^{m}\left(D_{1}\right)=$ $q_{1} D_{1}+\cdots+q_{m} D_{m}$, then $P(T)=T^{m}-q_{m} T^{m-1}-\cdots-q_{i}$ is the minimal polynomial of $T$ on this group of cones (considered as elements of $H_{n-1}\left(V_{t}-H_{\infty}, V_{t} H_{u_{0}}-H_{\infty}\right.$ ), cf. (3.17)).

Theorems I' and II' follow immediately from

(10.1) Theorem. $P(T)=\Pi\left(T^{m_{i}}-1\right)$. 
(10.2) Proof. The method of proof is to take a new hyperplane pencil $L^{\prime}$ with which we can express the homology of $V_{t} \cap H_{u}$ and hence the vanishing cycles $d_{i}$. There are two main cases, according as $s<n$ (i.e., $\operatorname{dim} M_{s}>0$ ) or $s=n$ (i.e., $x$ is one of the points of $M_{n}$ ).

B. Case 1. $s<n$. First we remark that the base section $u_{0}$ can be chosen close enough to 0 so as to meet any pre-assigned neighborhood of $x$, for instance, the domain of definition of the local coordinates $z_{1}, \cdots, z_{n}$, but not close enough to interfere with the $u(t), t$ small.

For convenience, let $L$ be given by $u=z_{1}+\cdots+z_{s}+z_{s+1}^{2}+\cdots+z_{n}^{2}$. The lack of higher powers will not affect the conclusions (see note 1 of $9 \mathrm{E}$ ). The new general pencil $L^{\prime}$ can be taken to be $z_{s+1}=$ const, since no member of it is tangent to $M_{s}$ near $x$.

(10.3) Proposition. (1) The pencil $L^{\prime}$ bas $2 m$ tangencies on $V_{t} \cap H_{u_{0}}$ near $x$.

(2) The $2 m$ branch points are arranged in two nonintersecting circles of $m$ points each (when $t$ is small), each moving as around a point of type $\left(m_{1}, \ldots\right.$, $m_{s}$ ) when $t$ goes about 0 .

(3) If the cuts in these two groups are chosen appropriately, then as $u$ approaches one of the $u(t)$, the cycle vanishing there is the sum of a cone from one group and a cone from the other.

(10.4) Proof. The tangencies of $L^{\prime}$ on $V_{t} \cap H_{u_{0}}$ depend on the places where $L^{\prime}$ is tangent to the singularities of $V_{0} \cap H_{u_{0}}$ (part 9E). Near $x$ there are only $s$ components for both $V_{0}$ and $V_{0} \cap H_{u 0}$; hence, there will be the singular subvarieties $M_{1}^{\prime}, \ldots, M_{s}^{\prime}$ on $V_{0} \cap H_{u_{0}}$ (analogous to the $M_{i}$ on $V_{0}$ ). In fact, $M_{i}^{\prime}=M_{i} \cap H_{u_{0}}$ and points in each are of the same type.

Now $H_{u}$ is not tangent to $M_{i}(i<s)$ near $x$, so no member of $i_{L}{ }^{\prime}$ can be tangent to $M_{i} \cap H_{u_{0}}$ (because $M_{i} \cap H_{u}$ is a pencil without singularities). But since $H_{0}$ cuts an ordinary double point on $M_{s}$, a nearby section, like $M_{s} \cap H_{u_{0}}$, will have two tangent planes in $L^{\prime}$ (part 8). Hence, for small $t$, there will be two groups of branch points on $V_{t} \cap H_{u_{0}}$.

Since $V_{0}=m_{1} V^{1}+\cdots+m_{s} V^{s}$ near $x, H_{0} \cap H=m_{1} V^{1} \cap H_{u_{0}}+\cdots+m_{s} V^{s}$ $\cap H_{u_{0}}$, so both these groups are of type $\left(m_{1}, \cdots, m_{s}\right)$ and there are $m$ points in each.

As $u_{0}$ approaches one of the $u(t)$, two branch points come together, one from each group, so the vanishing cycle is the sum of a cone from each group.

We can demonstrate (10.3) analytically by using the local equations for $L$ and $L^{\prime}$ : 
$V_{t}, H_{u}$ and $z_{s+1}=0$ meet in a singular variety when the Jacobian matrix $\partial\left(V_{t}, H_{u}, z_{s+1}\right) / \partial\left(z_{1}, \cdots, z_{n}\right)$ has rank $<3$.

Here, this Jacobian is

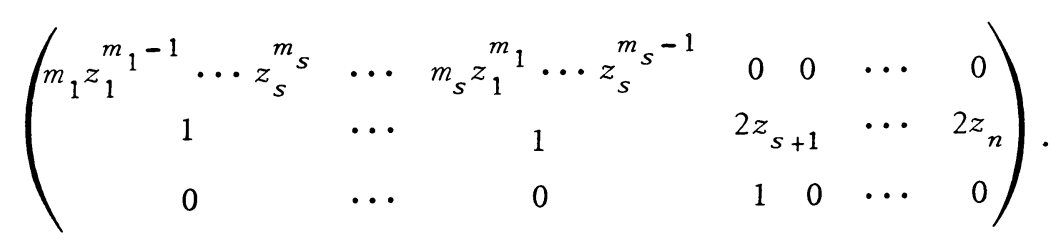

Since no $z_{i}=0(i=1, \cdots, s)$, the rank of this matrix is the same as that

$$
\left(\begin{array}{cccccccc}
m_{1} & m_{2} & \cdots & m_{s} & 0 & 0 & \cdots & 0 \\
z_{1} & z_{2} & \cdots & z_{s} & 2 z_{s+1} & 2 z_{s+2} & \cdots & 2 z_{n} \\
0 & 0 & \cdots & 0 & 1 & 0 & \cdots & 0
\end{array}\right) .
$$

Rank $<3$ means that $z_{i}=\lambda m_{i}(i=1, \cdots, s)$ and $z_{s+2}=\cdots=z_{n}=0$. Hence, $u=m \lambda+z_{s+1}^{2}$ and $t=c \lambda^{m}, c \neq 0$. Hence, the branch points $z_{s+1}$ on $V_{t} \cap H_{u}$ are given by $z_{s+1}=\left(u-c^{\prime} t^{1 / m}\right)^{1 / 2}, c^{\prime} \neq 0$.

The assertions of (10.3) follow easily from the simple nature of this algebraic function.

C. We apply (10.3) to prove (10.1).

Consider the boundary map

$$
\partial: H_{n-1}\left(V_{t}, V_{t} \cap H_{u_{0}}\right) \rightarrow H_{n-2}\left(V_{t} \cap H_{u_{0}}, V_{t} \cap H_{u_{0}} \cap H_{v_{0}}^{\prime}\right)
$$

where $H_{v_{0}}^{\prime}$ is in $L^{\prime}$. Better still, consider the same map on the relative groups of the corresponding affine varieties (i.e., with the hyperplane at infinity removed).

By (3.17) the cones we have been considering are free generators of their respective groups. We have $P(T) D_{1}=0$. Applying $\partial$ gives $P(T) d_{1}=0$. But (10.3) tells us that $d_{1}$ is the sum of cones on which, by induction, the P-L transformation has minimal polynomial $Q(T)=\prod\left(T^{m_{i}}-1\right)$.

Hence, $Q(T)$ divides $P(T)$, but since they are both monic and of the same degree $m$, they must be equal.

D. Case 2. $s=n$. In this case there will be no nice form for the new pencil $L^{\prime}$, since we have run out of coordinates. The best we can do is let $L$ be $u=z_{1}+\cdots+z_{n}$, as before, and $L^{\prime}: v=c_{1} z_{1}+\cdots+c_{n} z_{n}$, with distinct nonzero constants $c_{i}$.

Let $V_{0}=m_{1} V^{1}+\cdots+m_{n} V^{n}$ near $x$. We need to know the tangencies of $L^{\prime}$ on $V_{t} \cap H_{u_{0}}$.

(10.5) Proposition. (1) $L^{\prime}$ bas $(n-1) m$ tangencies on $V_{t} \cap H_{u_{0}}$ near $x$.

(2) These $(n-1) m$ branch points are arranged in $n$ nonintersecting circles 
of $m-m_{i}$ points each (when $t$ is small), eacb moving as around a point of type $\left(m_{1}, \ldots, \hat{m}_{i}, \cdots, m_{n}\right)$ when $t$ goes around 0 .

(10.6) Proof. As in part B we examine the singular subvarieties of $V_{0} \cap$ $H_{u_{0}}$. Since this is $(n-2)$-dimensional, we have the partition (near $\left.x\right) V_{0} \cap H_{u_{0}}$ $=M_{1}^{\prime} \cup \ldots \cup M_{n-1}^{\prime}$ where $M_{i}^{\prime}=M_{i} \cap H_{u u_{0}}$. As before, the $H_{u}(u$ small) are not tangent to $M_{1}, \cdots, M_{n-1}$, so $L^{\prime}$ will not have tangents on $M_{1}^{\prime}, \ldots, M_{n-2}^{\prime}$ near $x$ (because their dimension is $>0$ and $L^{\prime}$ is general).

But $M_{n-1}^{\prime}$ is a set of points, so the members of $L^{\prime}$ we seek a re the ones that contain a point of $M_{n-1}^{\prime}$.

Let $p_{i}$ be the point $\left(0 \cdots u_{0} \cdots 0\right)$, with $u_{0}$ in the $i$ th coordinate. This is where $V^{1}, \cdots, \hat{V}^{i}, \ldots, V^{n}, H_{u_{0}}$ meet near $x$. Now $V_{0} \cap H_{u_{0}}=m_{1}\left(V^{1} \cap H_{u_{0}}\right)$ $+\cdots+m_{n}\left(V^{n} \cap H_{u_{0}}\right)$ and $M_{n-1}^{\prime}=\left\{p_{i}\right\}$. We now just remark that $p_{i}$ is of type $\left(m_{1}, \cdots, \hat{m}_{i}, \cdots, m_{n}\right)$ and so contributes a cycle of $m-m_{i}$ branch points.

Finally, the total number of branch points near $x$ is $\Sigma\left(m-m_{i}\right)=(n-1) m$.

The picture in the $v$-line $L^{\prime}$ looks like

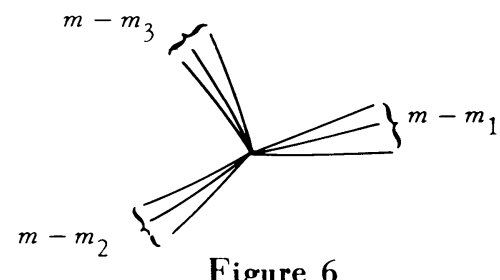

Figure 6

E. We must now examine the homology groups of the several varieties at hand. Since the question is local, the topology involved is the same as that of the affine pencil $z_{1}^{m_{1}} \cdots z_{n}^{m_{n}}=t$. The topology of $V_{t}$ is given by

(10.7) If $k$ is the g.c.d. of the $m_{i}$, then $V_{t}$ is homeomorphic to the sum of $k$ copies of $T^{n-1} \times R^{n-1}$, where $T^{n-1}$ is an $(n-1)$-torus and $R^{n-1}$ is Euclidean $(n-1)$-space. The $k$ pieces of $V_{t}$ permute cyclically as $t$ circles 0 .

(10.8) Proof. One just examines the map $\lambda: V_{t} \rightarrow R^{n-1}$ given by $\lambda\left(z_{1}, \cdots, z_{n}\right)=\left(\left|z_{1}\right|, \ldots,\left|z_{n}\right|\right)$ and observes that the image is homeomorphic to $R^{n-1}$ and each fibre is $k$ copies of $T^{n-1}$.

Now consider the exact sequence

$$
\begin{aligned}
& \text { (10.9) } H_{n-1}\left(V_{t} \cap H_{u_{0}}\right) \rightarrow H_{n-1}\left(V_{t}\right) \rightarrow H_{n-1}\left(V_{t}, V_{t} \cap H_{u_{0}}\right) \stackrel{\partial}{\rightarrow} \\
& H_{n-2}\left(V_{t} \cap H_{u 0}^{n}\right) \text {. }
\end{aligned}
$$

The first group is 0 (3.15), the second is $Z^{k}$ and the third is $Z^{m}$. Since the $D_{i}$ generate $H_{n-1}\left(V_{t}, V_{t} \cap H_{u 0}\right)$ and $\partial D_{i}=d_{i}$, the $d_{i}$ span a subgroup of rank $m-k$ in $H_{n-2}\left(V_{t} \cap H_{u_{0}}\right)$. In other words, there are just $k$ relations among them.

Let $P(T)$ be the minimal polynomial of $T$ on the $D$ 's and $Q(T)$ the minimal 
polynomial on the $d$ 's. Then $\operatorname{deg} p=m$ and $Q(T) \mid P(T)$. Now the minimal polynomial of $T$ on all the $(n-1) m$ cones in $V_{t} \cap H_{u_{0}}$ is

$$
\begin{aligned}
R(T) & =\underset{i}{1 . c \cdot m \cdot}\left(\left(T^{m} 1-1\right), \cdots,\left(T^{m_{j}}-1\right), \cdots,\left(T^{m} n_{-1}\right)\right) \\
& \bar{\prod}\left(T^{m_{i}}-1\right) /\left(T^{k}-1\right) .
\end{aligned}
$$

Then $Q(T) \mid R(T)$, so $\operatorname{deg} Q \leq \operatorname{deg} R=m-k$. If $\operatorname{deg} Q<m-k$, then all the $d$ 's would depend on the first $m-k-1: d_{1}, T\left(d_{1}\right), \ldots, T^{m-k-2}\left(d_{1}\right)$, a contradiction. Hence, $Q=R$.

Thus, $P=R S$ where $S$ is monic and of degree $k$.

Now observe that because the $k$ pieces of $V_{t}$ are disjoint and permuted cyclically by $T$, the cones $D_{i}, T\left(D_{i}\right), \ldots, T^{k-1}\left(D_{i}\right)$ are all disjoint for any $i$. Hence, when we look at $D\left(d_{m+1}, g_{m+1}\right)$ and bring $d_{m+1}$ back around each $g_{i}$ to calculate the coefficients of $P$, it will never involve a $D_{i}$ unless $i \equiv m(\bmod k)$. Hence, $P(T)$ is a polynomial in $T^{k}$, so $S(T)=T^{k} \pm 1$. To prove (10.1), we need $S(T)=T^{k}-1$.

Consider the loop $b$ which surrounds all $m$ branch points in $L$. After carrying $d_{m+1}=T^{m}\left(d_{1}\right)$ around $b$, it vanishes along $g_{1}$. Hence, it becomes $\pm d_{1}$ after traversing $b$. The following proposition is easily verified.

(10.10) Proposition. $d_{m+1}$ becomes $d_{1}$ if and only if $S(T)=T^{k}-1$.

Hence, we assume (10.10) holds in dimension $<n$ and allow $u$ to trace out $b$, keeping $t$ small. The $m$ points $M_{n-1}^{\prime}$ trace out disjoint circles on $M_{n-1}$, so the $m$ groups of branch points in $V_{t} \cap H_{u_{0}}$ merely rotate around $v=0$ but within each group come back to their original positions in a different order.

(10.11) Proposition. Each of the $m$ cycles undergoes the transformation $T^{-m}$.

(10.12) Proof. Let us look at the point $p_{n}=(00 \ldots 0 u)$ in $M_{n-1}^{\prime}(u)$. If $L^{\prime}$ is $v=c_{1} z_{1}+\cdots+c_{n-1} z_{n-1}+z_{n}, H_{v}^{\prime}$ goes through $p_{n}$ for $v=u$. Then we know by (9.12) that $v=u+c t^{1 /\left(m-m_{n}\right)}+\cdots, c$ depending on $u$.

We calculate $c(u)$ as follows:

On $H_{u}$, the pencil $V_{t} \cap H_{u}$ is $z_{1}^{m_{1}} \cdots z_{n-1}^{m_{n-1}}\left(u-z_{1}-\cdots-z_{n-1}\right)^{m_{n}}=t$ and $L^{\prime}$ becomes $v=u+\left(c_{1}-1\right) z_{1}+\cdots+\left(c_{n-1}-1\right) z_{n-1}$. In order to make the equation for $V_{t} \cap H_{u}$ have the simple form of (9.12), replace $z_{n-1}$ by $\bar{z}_{n-1}=$ $z_{n-1}\left(u-z_{1}-\cdots-z_{n-1}\right)^{m_{n} / m_{n-1}}$, so $V_{t} \cap H_{u}$ is given by $z_{1}^{m_{1}^{n}} \cdots$
$z_{n-2}^{m_{n}-2} \bar{z}_{n-1}^{m_{n-1}}=t$.

Hence, the linear part of $v$ is $u+\left(c_{1}-1\right) z_{1}+\cdots+\left(c_{n-2}-1\right) z_{n-2}+$ $\left(c_{n-1}-1\right) \bar{z}_{n-1}\left(u-z_{1}-\cdots-z_{n-1}\right)^{-m_{n} / m_{n-1}}$, or, when $t$ is small enough, 


$$
u+\left(c_{1}-1\right) z_{1}+\cdots+\left(c_{n-2}-1\right) z_{n-2}+\left(c_{n-1}-1\right) u^{-m_{n} / m}{ }_{n-1} \bar{z}_{n-1} .
$$

From (9.12) we get

$$
z_{i}=\left(m_{i} /\left(c_{i}-1\right)\right) \lambda+\cdots, \quad i=1, \cdots, n-2,
$$

and

$$
\bar{z}_{n-1}=\left(m_{n-1} /\left(c_{n-1}-1\right)\right)_{u}^{m_{n} / m} n-1 \lambda+\cdots .
$$

This means $v-u=m \lambda+\cdots$ and

$$
t=c \lambda^{m-m_{n-1}-m_{n}} \lambda^{m} n-1 u^{m}+\cdots=c \lambda^{m-m} n^{m} n+\cdots, \quad c \text { a constant. }
$$

Hence, $v=u+c^{\prime} u^{-m_{n} /\left(m-m_{n}\right)} t^{1 /\left(m-m_{n}\right)}+\cdots, c^{\prime}$ a constant, or $v=$ $u\left(1+c^{\prime}\left(u^{1 /\left(m-m_{n}\right)}\right)-m_{t}^{1 /\left(m-m_{n}\right)}+\cdots\right)$.

So as $u$ travels on $b$ ( $t$ fixed), the Puiseux series around $p_{n}$ gets multiplied by $\epsilon^{-m}$, where $\epsilon$ is a primitive $\left(m-m_{n}\right)$ th root of 1 .

Hence, the $m-m_{n}$ cones in this group undergo the transformation $T^{-m}$. Similarly for all the other groups, which proves (10.11).

Since, $d_{1}$ is a sum of these $(n-1) m$ cones, (10.10) implies that $P(T)=$ $\Pi\left(T^{m_{i}}-1\right)$, as desired.

11. Normal crossings at the base.

A. We now weaken the assumptions we have been working under to allow for a base locus. This means that the parameter curve $C$ is the projective line $P^{1}$, so that the $V_{t}$ are just a 1 -dimensional linear system on $W$. Any two members will meet in the same algebraic cycle $B$ of codimension 2 in $W$.

The singularities of $B$ coincide with the points where some $V_{t}$ has singularities at the base. This is the same as the set of points where two (nonsingular) $V_{t}$ are tangent. A singularity of $B$ is singular for a unique $V_{t}$, since we are still assuming that the general fibre is nonsingular.

The homeomorphisms between the nonsingular $V_{t}$ induced by the local retractions clearly leave $B$ point-wise fixed and the same holds for any homology cycle that can be put in $B$. This, of course, is why there is no action in homology (outside of the middle dimension) when the $V_{t}$ are hyperplane sections.

In theory, one can eliminate the base by the following device:

Our family $V_{t}$ is given by the rational map $f: W \rightarrow P^{1}$. Let $W^{\prime}$ be the graph of $f$ in $W \times P^{1}$. Then we have the rational maps $f^{\prime}: W^{\prime} \rightarrow P^{1}$ and $g: W^{\prime}$ $\rightarrow W$ induced by the projections. We have

(11.1) (1) $f^{\prime}$ and $g$ are everywhere defined.

(2) $f \circ g=f^{\prime}$.

(3) $g$ is a biregular isomorphism between $W^{\prime}-g^{-1}(B)$ and $W-B$.

(4) If $t \in P^{1}$, then $g \mid f^{\prime-1}(t)$ is an isomorphism of $f^{\prime-1}(t)$ with $V_{t}$. 
By (3), the only singularities of $W^{\prime}$ lie in $g^{-1}(B)$, so if $W^{\prime \prime}$ is the desingularization (Hironaka) of $W^{\prime}$ obtained by blowing up pieces of $g^{-1}(B)$ and if $g^{\prime}$ : $W^{\prime \prime} \rightarrow W^{\prime}$ is the associated map, we get a rational map $f^{\prime} \circ g^{\prime}=f \circ g \circ g^{\prime}$ which is defined everywhere on $W^{\prime \prime}$ and gives us a pencil $V_{t}^{\prime}$ without base. If we consider the map $g^{\circ} g^{\prime}: W^{\prime \prime} \rightarrow W$, then $g^{\circ} g^{\prime}: V_{t}^{\prime} \rightarrow V_{t}$ is a blowing-up of $V_{t}$ along pieces of $B$.

This means that the map $H_{*}\left(V_{t}^{\prime}\right) \rightarrow H_{*}\left(V_{t}\right)$ induced by $g \circ g^{\prime}$ is surjective. Hence, if the main theorems hold for $V_{t}^{\prime}$, they hold for $V_{t}$.

B. Still, it may be worthwhile to examine the pencil $V_{t}$ directly when the base $B$ is particularly simple. This is useful for hypersurfaces in projective space, where a base locus cannot be avoided.

As before, we assume the singular fibre $V_{0}$ has only normal crossings. We will also assume that the pencil $V_{t}$ is transversal at the base. This just means that the components of $V_{0}$ and any one of the other $V_{t}$ are transversal wherever they meet, i.e., the ir local equations form a partial system of coordinates. This implies that only $V_{0}$ has singularities on $B$.

Analytically, this means that if $x \in B$ and $V^{1}, \ldots, V^{s}$ are the components of $V_{0}$ through $x$ with local equations $z_{1}, \cdots, z_{s}$ and multiplicities $m_{1}, \cdots$, $m_{s}$, then $s<n$ and there is another local coordinate $z_{s+1}$ such that $V_{t}$ is given by $z_{1}^{m_{1}} \cdots z_{s}^{m_{s}}=t z_{s+1}$.

Hence, the base (near $x$ ) is given by

$$
\left(z_{1}=z_{s+1}=0\right) \cup\left(z_{2}=z_{s+1}=0\right) \cup \cdots \cup\left(z_{s}=z_{s+1}=0\right) .
$$

Hence, $B$ consists of normal crossings, i.e., $V_{t}(t \neq 0)$ is not tangent to any $M_{i}$. Let $B_{i}=M_{i} \cap B$.

C. We now examine $V_{0}^{*}$. (9.4) and (9.6) yield

(11.2) Proposition. Let $H_{u}$ be a general pencil of byperplanes. Then each $H_{u}$ is tangent to at most one of the $M_{i}$ or $B_{i}$ and cuts an ordinary double point there. Also, if $H_{u}$ is tangent to $\bar{M}_{i}\left(\right.$ or $\left.\bar{B}_{i}\right)$, it is tangent to $M_{i}\left(\right.$ resp. $\left.B_{i}\right)$ but not at $B$.

The branch function and P-L transformation for tangencies of $H_{u}$ with $M_{i}$ were given by (9.12). For a tangency at $B$, we have

(11.3) Proposition. If $x \in B_{s}$ and $V_{t}$ is $z_{1}^{m_{1}} \cdots z_{s}^{m_{s}}=t z_{s+1}$ near $x$ and if some $H_{u}$ is tangent to $B_{s}$ at $x^{s}$, then $x^{t}$ is on the branch curve and the branch function is given by $u=$ Puiseux series in $t^{1 /(m-1)}, m=m_{1}+\cdots+m_{s}$.

(11.4) Proof. We can take $H_{u}$ to be $z_{1}+\cdots+z_{s+1}=u$. The Jacobian is 


$$
\left(\begin{array}{ccccccc}
m_{1} t z_{s+1} / z_{1} & \cdots & m_{s} t z_{s+1} / z_{s} & -t & 0 & \cdots & 0 \\
1 & \ldots & 1 & 1 & 0 & \cdots & 0
\end{array}\right) .
$$

When the rank is $<1$, we find $z_{i}=m_{i} z_{s+1}(-1)(i=1, \cdots, s)$ or $c z_{s+1}^{m}=$ $t z_{s+1}$ and $u=-(m-1) z_{s+1}$, from which (11.3) follows.

Hence, for each tangency on $B_{s}$ we have a cycle of $m-1$ branch points, so the P-L transformation on this group is a polynomial of degree $m-1$. By using an auxiliary pencil $H_{v}^{\prime}$ and calculating the cones of $V_{t} \cap H_{u_{0}}$, we arrive at

(11.5) Theorem. The minimal polynomial for the $P-L$ transformation is $P(T)$ $=\Pi\left(T^{m_{i}}-1\right) /(T-1)$.

(11.6) Proof. As in part 10, there are two cases, according as $s<n-1$ or $s=n-1$.

First, observe that (locally) $V_{t}(t \neq 0)$ is homeomorphic to a $(2 n-2)$-ball, so in the exact sequence (10.9), $\partial$ is injective. Hence, the minimal polynomial of $T$ on $d_{1}=\partial D_{1}$ is also $P(T)$. We find $d_{1}$ by looking at the tangencies of $H_{v}^{\prime}$ on $V_{t} \cap H_{u_{0}}$ near $x$.

Case 1. $s<n-1$. This is quite analogous to (10.3). $H_{0} \cap B_{s}$ has an ordinary double point at $x$, so for two (small) values of $v, H_{v}^{\prime}$ will be tangent to $H_{u_{0}} \cap B_{s}$ near $x$. Hence, we have two groups of $m-1$ branch points on $V_{t}$ $\cap H_{u_{0}}$ from which $d_{1}$ is formed. By induction, the minimal polynomial of $T$ on these $2 m-2$ cones is $P(T)$. Hence, the same is true for $d_{1}$ (since we know its minimal polynomial has degree $m-1$ ) and $D_{1}$.

Case 2. $s=n-1 . V_{0}$ is locally $V^{1} \cup \cdots \cup V^{n-1}$ and $B$ is $\left(V^{1} \cap V_{t}\right) \cup$ $\cdots \cup\left(V^{n-1} \cap V_{t}\right)$. Hence, $V_{0} \cap H_{u_{0}}$ is $\left(V^{1} \cap H_{u_{0}}\right) \cup \cdots \cup\left(V^{n-1} \cap H_{u_{0}}^{t}\right)$. and the base of the pencil $V_{t} \cap H_{u_{0}}$ is $\left(V^{1} \cap V_{t} \cap H_{u_{0}}\right) \cup \cdots \cup\left(V^{n-1} \cap V_{t}\right.$ $\left.\cap H_{u_{0}}\right)$. Hence, the tangencies of $H_{v}^{\prime}$ on $V_{t} \cap H_{u_{0}}$ near $x$ are of two kinds:

(11.7) (1) A group of $m$ cones with minimal polynomial $\Pi\left(T^{m_{i}}-1\right)$ coming from the non-base-point $\left(V^{1} \cap H_{u_{0}}\right) \cap \ldots \cap\left(V^{n-1} \cap H_{u_{0}}\right)$.

(2) $n-1$ groups of $m-m_{i}-1$ cones each, coming from the basepoint $\left(V^{1} \cap V_{t} \cap H_{u 0}\right) \cap \ldots \cap\left(\widehat{V^{i} \cap V_{t} \cap H_{u 0}} \cap \ldots \cap\left(V^{n-1} \cap V_{t} \cap H_{u_{0}}\right)\right.$.

The minimal polynomial of $T$ on these $(m-1)(n-1)$ cones is $\Pi\left(T^{m_{i}}-1\right)$. Since the minimal polynomial on $d_{1}$ is of degree $m-1$, it can only be $\Pi\left(T^{m_{i}}-1\right) /(T-1)=P(T)$ or $\Pi\left(T^{m_{i}}-1\right) /(T+1)$. We shall eliminate the latter possibility.

First we have a result analogous to (10.10).

(11.8) Proposition. Let $b$ be a (counterclockwise) loop surrounding the $m-1$ branch points. Then $b$ induces the transformation $T^{-(m-1)}$ if and only if the minimal polynomial is $P(T)$. 
Now to examine the action of $b$ on the $(m-1)(n-1)$ cones of $V_{t} \cap H_{u_{0}}$ we have two cases, according as the cone is induced by a base-point or not (cf. $(11.7))$.

$V_{t}$ is given by $z_{1}^{m_{1}} \cdots z_{n-1}^{m_{n-1}}=t z_{n}, L$ by $u=z_{1}+\cdots+z_{n}$ and $L^{\prime}$ by $v=$ $c_{1} z_{1}+\cdots+c_{n} z_{n}$ (higher powers will not affect the results). Consider first the base-point $(u, 0, \cdots, 0)$ on $V_{t} \cap H_{u}$ (as $u$ traverses $\left.b\right) . z_{2}, \cdots, z_{n}$ are local coordinates there and we have

$$
\begin{aligned}
V_{t} \cap H_{u}: & \left(u-z_{2}-\cdots-z_{n}\right)^{m} 1_{z_{2}}^{m} 2 \cdots z_{n-1}^{m}=t z_{n}, \\
L^{\prime}: & v=c_{1} u+\left(c_{2}-c_{1}\right) z_{2}+\cdots+\left(c_{n}-c_{1}\right) z_{n^{\bullet}}
\end{aligned}
$$

In order to put the local equation of $V_{t} \cap H_{u}$ into the standard form, let $\bar{z}_{2}=z_{2}\left(u-z_{2}-\cdots-z_{n}\right)^{m_{1} / m_{2}}$, so (11.9) becomes

$$
\begin{gathered}
V_{t} \cap H_{u}: \bar{z}_{2}^{m} \cdots z_{n-1}^{m}=t z_{n}, \\
L^{\prime}: v-c_{1} u=\left(c_{2}-c_{1}\right) u^{-m_{1} / m_{2}} \bar{z}_{2}+\left(c_{3}-c_{1}\right) z_{3}+\cdots+\left(c_{n}-c_{1}\right) z_{n}+\text { h.p. }
\end{gathered}
$$

By (11.3), the branch points on $V_{t} \cap H_{u}$ are given by $v-c_{1} u=$ const $u^{-m_{1} /\left(m-m_{1}-1\right)} t^{1 /\left(m-m_{1}-1\right)}+\cdots$ where the constant does not depend on $u$. This can be written

$$
v=u\left(c_{1}+\text { const } u^{-(m-1) /\left(m-m_{1}-1\right) 1 /\left(m-m_{1}-1\right)}+\cdots\right) .
$$

We conclude that as $u$ traverses $b$, the center of this group of $m-m_{1}-1$ branch points makes one circuit and its members undergo the permutation $T^{-(m-1)}$. Similarly for the other $n-2$ groups of $m-m_{i}-1$ points. As for the non-basepoint $(0, \cdots, 0, u)$, we eliminate $z_{n}$ to get

$$
\begin{aligned}
V_{t} \cap H_{u}: & z_{1}^{m}{ }_{1} \cdots z_{n-1}^{m} n_{n-1}=t\left(u-z_{1}-\cdots-z_{n-1}\right), \\
L^{\prime}: & v=c_{n} u+\left(c_{1}-c_{n}\right) z_{1}+\cdots+\left(c_{n-1}-c_{n}\right) z_{n-1} \cdot
\end{aligned}
$$

Then from (9.12) (or directly) we find the branch points on $L^{\prime}$ to be $v=$ $c_{n} u+$ const $(t u)^{1 / m}=u\left(c_{n}+\right.$ const $\left.u^{-(m-1) / m} t^{1 / m}+\cdots\right)$. Hence, this group behaves just like the other $n-1$ groups of cones, and the effect of traversing $b$ is $T^{-(m-1)}$. Q.E.D.

\section{Examples of Picard-Lefschetz transformations.}

A. We give here a few examples of the P-L transformation for certain simple pencils and singularities.

The first case to consider is where the $V_{t}$ are curves. The possible types 
of singular fibres have been classified by Kodaira [3] for genus 1 and Ogg [5] for genus 2. Kodaira worked out the P-L transformation using the modular function, but it can also be done with the previously described theory.

B. Example 1. $V_{0}=3$ rational lines meeting at a point. This singularity was analyzed in part 7B. We get six cones in $H_{1}\left(V_{t}, V_{t} \cap H_{u}\right)$ and $A \rightarrow B \hookrightarrow C$ $\rightarrow A$ under $T$. Also $\partial A=\partial B=\partial C$ since $T$ is the identity on $V_{t} \cap H_{u}$. Hence $A-B$ and $B-C$ form a basis for $H_{1}\left(V_{t}\right)$ and we have the matrix $\left(\begin{array}{rr}0 & 1 \\ -1 & -1\end{array}\right)$ of order 3 .

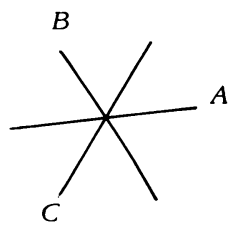

Figure 7

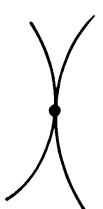

Figure 8

Example 2. $V_{0}=2$ rational lines tangent at a point. One could blow up the singularity and analyze the resulting normal crossings (viz. $11 \mathrm{~A}$ ), but it is simpler to treat it directly.

Locally, $V_{t}$ is $x\left(x+y^{2}\right)=t$ and $H_{u}$ is $u=x+y$. The Jacobian is

$$
\left(\begin{array}{cc}
2 x+y^{2} & 2 x y \\
1 & 1
\end{array}\right),
$$

so the branch curve is $2 x+y^{2}-2 x y=0$. This is nonsingular at $(0,0)$ and $x=-1 / 2 y^{2}+\cdots, u=y+\cdots, t=-1 / 4 y^{4}+\cdots$. Hence, the branch function is essentially $u=c t^{1 / 4}, c \neq 0$. Under $T$ we have $A \rightarrow B \rightarrow C \rightarrow D$ and since all the boundaries are equal, $T(D)=2 D-2 C+2 B-A \cdot H_{1}\left(V_{t}\right)$ is spanned by $A-B$ and $B-C$.

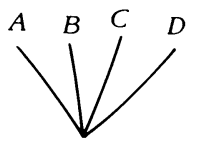

Figure 9

$T(A-B)=B-C$ and $T(B-C)=C-D$. Since $(A-B, B-C)=(B-C, C-D)$ $=-(C-D, B-C)$, we have $T(B-C)=-(A-B)$ and the matrix is $\left(\begin{array}{rr}0 & 1 \\ -1 & 0\end{array}\right)$.

Example 3. $V_{0}=s$ rational lines forming a closed "polygon." Each intersection is an ordinary double point, so we have $s$ pairs of cones with

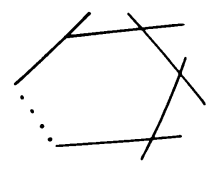

Figure 10

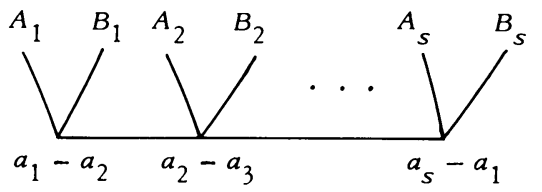

Figure 11

$\partial A_{1}=\partial A_{2}=a_{1}-a_{2}$, etc. We can assume $\partial A_{s}=a_{s}-a_{1}$ by choosing the path correctly, because $V_{t}$ is irreducible. Here, $H_{1}\left(V_{t}\right)$ is spanned by $\Sigma A_{i}$ and 
$B_{1}-A_{1}$. Since for all $i, B_{i}-A_{i}=B_{1}-A_{1}$ in $H_{1}\left(V_{t}\right)$, the matrix is $\left(\begin{array}{ll}1 & s \\ 0 & 1\end{array}\right)$.

Example 4. $V_{0}$ is like Figure 12, where each component is a rational curve, four of multiplicity 1 and $s+1$ of multiplicity 2. The cones look like Figure 13 (we have ignored two sets of three). Underneath the first

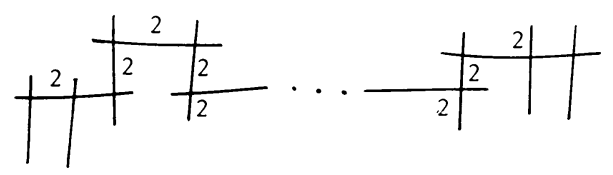

Figure 12

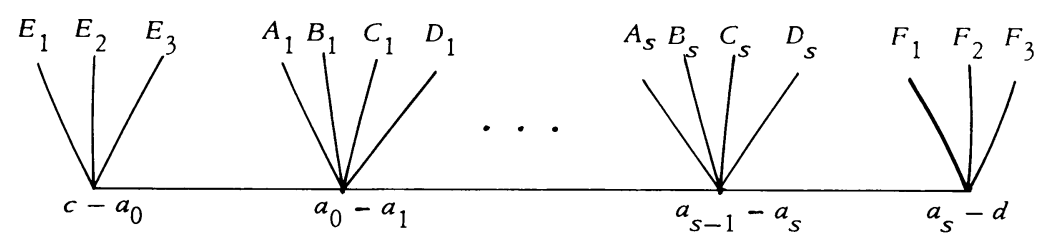

Figure 13

member of each group is its boundary, e.g., $\partial E_{1}=c-a_{0}, \partial A_{1}=a_{0}-a_{1}$, etc. The boundaries of the other cones are determined by the points $c, d, a_{i}, b_{i}(i=$ $0, \cdots, s)$ of $V_{t} \cap H_{u}$ where $T(c)=c, T(d)=d, T\left(a_{i}\right)=b_{i}$ and $T\left(b_{i}\right)=a_{i}$.

Let $G=\Sigma\left(A_{i}-B_{i}\right)+E_{1}-E_{2}+F_{1}-F_{2}$ and $H=A_{1}-C_{1} . G$ and $H$ span $H_{1}\left(V_{t}\right)$ since $(G, H)=1$. We have $B_{1}-D_{1}=-A_{1}-C_{1}$ and $E_{1}-E_{3}=F_{1}-F_{3}$ $=0$, so the P-L matrix is $\left(\begin{array}{rr}-1 & s \\ 0 & -1\end{array}\right)$.

C. The P-L transformation for elliptic curves can be calculated more easily after expressing the general $V_{t}$ as a 2 -sheeted covering of the projective line, since the corresponding $g_{2}^{1}$ suffices to calculate $H_{1}\left(V_{t}\right)$. Of course, this is also true for any hyperelliptic pencil. This can be written in the form

(12.1) $y^{2}=f(x, t), f$ a polynomial of degree $2 g+1$ or $2 g+2$ in $x(g=$ genus of $\left.V_{t}\right)$.

The cones and their behavior can be calculated with respect to the pencil $x=$ const. The only difficulty is that (12.1) may represent a singular surface in $P^{3}$, so must be desingularized if one wants to see what the "real" fibre $V_{0}$ is. This, of course, does not change the P-L transformation (cf. 11A).

Example 5. Consider the pencil $y^{2}=x^{3}+x^{2}+t^{s}, s>1$. The point $(0,0,0)$ is singular, but after a finite number of quadratic transformations (in fact after $[s / 2])$, the singularity is resolved and the new $V_{0}$ is just the curve of Example 3. Observe that as $t$ moves around 0 once, $t^{s}$ moves around $s$ times. In other words the P-L transformation is the $s$ th power of the one corresponding to the pencil $y^{2}=x^{3}+x^{2}+t$. But $V_{0}$ here just has an ordinary double point, so the matrix is $\left(\begin{array}{ll}1 & 1 \\ 0 & 1\end{array}\right)$, and its sth power is $\left(\begin{array}{ll}1 & s \\ 0 & 1\end{array}\right)$, as before. 
Example 6. Consider Ogg's example: $y^{2}=x^{5}-t^{s}$. As $s$ changes, the fibre $V_{0}$ on the nonsingular surface assumes various shapes, e.g., $s=2$ and $s$ $=3$ are represented in Figures 14 and 15 (the components are rational lines with their corresponding multiplicities).

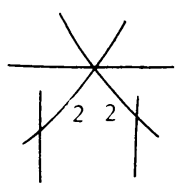

Figure 14

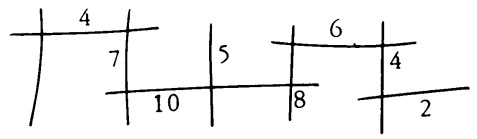

Figure 15

But as was explained above, the P-L transformation is just the sth power of the one for the pencil $y^{2}=x^{5}-t$. To calculate the latter we use the pencil $x=u$ and find two groups of branch points and cones, viz., $u=t^{1 / 5}$ and $u=\infty$. They are represented in Figure 16.

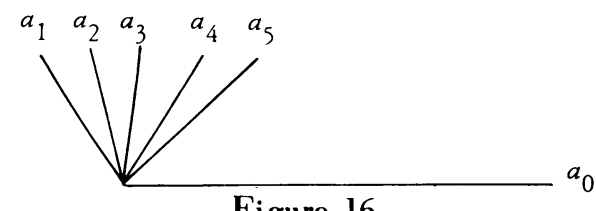

Figure 16

There is one relation in $H_{1}\left(V_{t}, V_{t} \cap H_{u}\right)$ (cf. (3.11)), viz.,

$$
a_{0}-a_{1}+a_{2}-a_{3}+a_{4}-a_{5}=0 \text {. }
$$

Furthermore, under $T$ we have

$a_{0} \rightarrow a_{0}, \quad a_{1} \rightarrow a_{2} \rightarrow a_{3} \rightarrow a_{4} \rightarrow a_{5}, a_{5} \rightarrow 2 a_{5}-2 a_{4}+2 a_{3}-2 a_{2}+a_{1}$.

If we put $A=a_{1}-a_{2}, B=a_{2}-a_{3}, C=a_{3}-a_{4}, D=a_{4}-a_{5}$, then $A, B$, $C$ and $D$ span $H_{1}\left(V_{t}\right)$ and the matrix is the companion matrix to the polynomial $x^{4}-x^{3}+x^{2}-x+1$, so is of order 10 .

More generally, we have the following:

(12.4) Proposition. Let $y^{2}=x^{m}-t$. If $m$ is odd, the matrix is companion to $\left(x^{m}+1\right) /(x+1)$, so is of order $2 m(m>1)$. If $m$ is even, the matrix is companion to $\left(x^{m}-1\right) /\left(x^{2}-1\right)$, so is of order $m(m>2)$.

D. Example 7. $V_{t}$ = quadrics in projective space $P_{n} \cdot H_{n-1}\left(V_{t}\right)=0$ if $n$ is even, so the P-L transformation is trivial. Assume $n=2 m+1$. Then $H_{n-1}\left(V_{t}\right)$ (for $V_{t}$ nonsingular) is generated by two linear $m$-spaces $A$ and $B$ with the following intersection properties:

$$
\begin{array}{lll}
(A, A)=(B, B)=0, & (A, B)=1 \quad \text { if } m \text { is odd } \\
(A, A)=(B, B)=1, & (A, B)=0 \quad \text { if } m \text { is even. }
\end{array}
$$


By (3.7) the self-intersection number of the cycle vanishing at an ordinary double point is $2(-1)^{m}$. Since it must be of the form $r A+s B$ for some integers $r$ and $s$, (12.5) shows that the vanishing cycle must be $A-B$. From this it follows that the P-L matrix is $\left(\begin{array}{ll}0 & 1 \\ 1 & 0\end{array}\right)$.

In the space of dimension $n(n+3) / 2$ which parametrizes the quadrics of $P_{n}$, the singular quadrics form a hypersurface of degree $n+1$. Hence, if the intersection multiplicity of the pencil $V_{t}$ with this hypersurface at $V_{0}$ is even, the $\mathrm{P}-\mathrm{L}$ transformation is the identity and $\left(\begin{array}{ll}0 & 1 \\ 1 & 0\end{array}\right)$ otherwise.

The intersection multiplicity can be calculated as follows: choose projective coordinates so that one $V_{t}$ has equation $x_{0}^{2}+\cdots+x_{n}^{2}=0$. Then the multiplicity of $V_{0}$ is the number of times 0 counts as an eigenvalue of the symmetric matrix representing $V_{0}$. For instance, if the singular set of $V_{0}$ is $r$-dimensional and $V_{t}$ is not tangent to it, the multiplicity is $r+1$.

Example 8. $V_{t}=$ cubic surfaces in $P_{3} \cdot H_{2}\left(V_{t}\right)$ is generated by the lines on $V_{t}$. The set of all these lines forms a surface of degree 9 , since the nonsingular $V_{t}$ contain just 27 lines. Hence, when $t$ makes a loop, the 27 lines undergo some permutation, and we conclude that

(12.6) The P-L transformation for cubic surfaces in $P_{3}$ is of finite order.

For instance, as a simple special case we have

(12.7) If $V_{0}=3$ planes and $V_{t}$ is otherwise general, the P-L transformation is the identity.

We can see this as follows: the pencil $V_{t} \cap H_{u}$ near $V_{0} \cap H_{u}$ is of the type of Example 3 with $s=3$, so there is a single invariant vanishing cycle $b$. Now $V_{0}$ has 3 double lines and 1 triple point. The former yield 9 double points at the base, which result in 9 invariant cones on $V_{t}$ (see (11.5)). Their boundaries must be $b$. The triple point yields three cones: $A, T A, T^{2} A$, with boundaries $a, a+3 b, a+6 b$, where $(a, b)=-1$. The relation among these 12 cones induced by $b$ (viz. 3D) is $(T-1)^{2} A=0$, so every cycle in $V_{t}$ is invariant.

Example 9. Finally, for an example of a less trivial transformation, we mention the case of quartic surfaces in $P_{3}$, with $V_{0}=4$ planes. $V_{0}$ has 6 double lines and 4 triple points, so we get 24 invariant cones plus 4 sets of 3 on which the minimal polynomial is $(T-1)^{3}$.

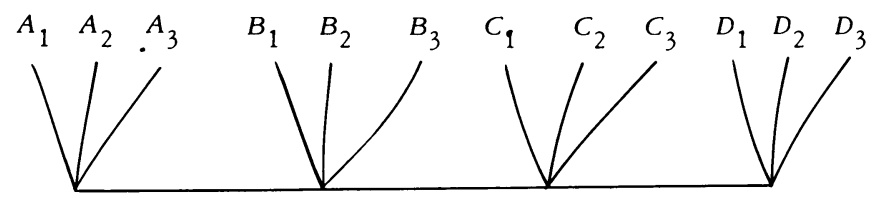

Figure 17 
$(T-1)^{2} A_{1},(T-1)^{2} B_{1}$, etc., are vanishing cycles which turn out to be homologous to one cycle $E$, while $F=A_{1}+B_{1}+C_{1}+D_{1}$ is a cycle such that $(E, F)=-1$. Hence, $(T-1)^{2} F=4 E \neq 0$, and we conclude

(12.8) The minimal polynomial of the P-L transformation on $H_{2}\left(V_{t}\right)$ is $(T-1)^{3}$.

\section{REFERENCES}

1. I. Fáry, Cohomologie des variétés algébriques, Ann. of Math. (2) 65 (1957), 2173. MR 18, 822 .

2. H. Hironaka, Resolution of singularities of an algebraic variety over a field of characteristic zero. I, II, Ann。 of Math. (2) 79 (1964), 109-326. MR 33 \#7333.

3. K. Kodaira, On compact analytic surfaces. II, Ann. of Math. (2) 77 (1963), 563626. MR $32 \# 1730$.

4. S. Lefschetz, L'analysis situs et la géométrie algébrique, Gauthier-Villars, Paris, 1924.

5. A. P. Ogg, On pencils of curves of genus two, Topology 5 (1966), 355-362. MR $34 \# 1321$.

6. E. Picard and G. Simart, Théorie des fonctions algébriques de deux variables indépendantes. Vols. I, II, Gauthier-Villars, Paris, 1897, 1906.

7. A. H. Wallace, Homology the ory on algebraic varieties, Internat. Series of Monographs on Pure and Appl. Math。, vol。6, Pergamon Press, New York, 1958. MR 20 \#46.

8. O. Zariski, A theorem on the Poincaré group of an algebraic hypersurface, Ann. of Math. (2) 38 (1937), 131-141.

9. A. B. Žižčenko, Homology groups of algebraic varieties, Izv. Akad. Nauk SSSR Ser. Mat. 25 (1961), 765-788. MR $25 \# 83$.

\section{APPENDIX AND SUPPLEMENTARY BIBLIOGRAPHY (PHILLIP A. GRIFFITHS)}

1. Restatement of the mondromy theorem. Let $D=\{t \in \mathrm{C}:|t|<1\}$ be the unit disc in $\mathrm{C}, X$ a complex manifold of dimension $n+1$ which admits a projective embedding, and $f: X \rightarrow D$ a proper, holomorphic mapping which has $t=0$ as the only critical value. Then $V_{t}=f^{-1}(t)$ is a smooth, projective variety for $t \neq 0$, and by Hironaka we may assume that $V_{0}$ has normal crossings. This is the localization in the parameter space of the situation $f: W \rightarrow C$ of the above paper. We write

$$
V_{0}=m_{1} D_{1}+\cdots+m_{l} D_{l}
$$

where the $D_{j}$ are smooth divisors meeting transversely. We then define

$$
F_{s}\left(V_{0}\right)=\sum_{i_{1}<\cdots<i_{s}} D_{i_{1}} \cap \cdots \cap D_{i_{s}},
$$

so that the following hold: 


$$
\begin{aligned}
& F_{1}\left(V_{0}\right)=\left|V_{0}\right| \text { is the support of } V_{0} ; \\
& F_{s+1}\left(V_{0}\right) \subset F_{s}\left(V_{0}\right) ; \text { and } \\
& F_{s}\left(V_{0}\right)=\text { points of order } s \text { on }\left|V_{0}\right| .
\end{aligned}
$$

The two numerical characters

$$
\left\{\begin{array}{l}
N=1 . c \cdot \text { m. }\left(m_{1}, \cdots, m_{l}\right), \\
r_{0}=\max _{s}\left\{F_{s}\left(V_{0}\right) \neq \varnothing\right\} \leq n+1
\end{array}\right.
$$

are associated to the situation $f: X \rightarrow D$.

Fix $t_{0} \neq 0$ and set $V=V_{t_{0}}, H^{q}(V)=H^{q}(V, \mathbf{Q})$, and denote by $T: H^{q}(V) \rightarrow$ $H^{q}(V)$ the Picard-Lefschetz transformation given by the generator of $\pi_{1}(D-\{0\})$ acting on the cohomology $H^{q}(V)$. Theorems $\mathrm{I}^{\prime}$, $\mathrm{II}^{\prime}$ of the paper may be summarized by the matrix equation

$$
\left(T^{N}-I\right)^{r}=0 \quad\left(r_{1}=\min \left(r_{0}, q+1\right)\right) .
$$

This result is generally referred to as the monodromy theorem. Since Landman's thesis there have been several proofs of (A.2) of widely varying natures, and our purpose is to present a short bibliography to these proofs.

2. Geometric proofs. These have been given by Clemens [11] and DeligneGrothendieck [13]. The idea is to consider the situation

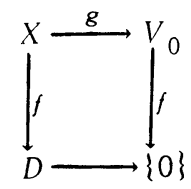

where $g$ is the retraction of $X$ onto the singular fibre $V_{0}$. Thus $g: V_{t} \rightarrow V_{0}$ may be viewed as a sort of "collapsing" map. By using the normal crossings of $V_{0}$, we may assume that $f \circ g=f$. If we now apply the Leray spectral sequence to $X \stackrel{g}{\rightarrow} V_{0}$, then $f$ operates in this via its operation on the Leray direct image sheaves $R_{g_{*}}^{q}(\mathbf{Q})$. The monodromy theorem follows by examining this action. A further discussion of this is given in $\$ 15$ of [14].

There is also a local version of the monodromy theorem when the fibres $V_{t}$ are hypersurfaces in $\mathrm{C}^{n+1}$ acquiring an isolated singular point. Early results here by Pham, Brieskorn, and Milnor led to a fascinating interplay between the P-L transformation and exotic spheres [16].

3. Proofs using the Picard-Fuchs equation. The sheaf $\mathcal{H}^{q}=R_{f_{*}}^{q}(\mathbf{C}) \otimes_{\mathbf{C}} \Theta_{D}$ admits a canonical flat connection $\nabla$, the Gauss-Manin connection, such that 
$R_{f_{*}}^{q}(\mathrm{C})$ forms the subsheaf of flat sections. Now $\mathcal{H}^{q}$ extends to a coherent sheaf on $D$, but $\nabla$ will generally have a singularity at $t=0$ whose "residue" is $\log T$. The detailed study of $\left(\mathcal{H}^{q}, \nabla\right)$ led Brieskorn [10], Deligne [12], and Katz [15] to a proof of the monodromy theorem. Moreover, Katz was able to ref ine the index of unipotency $r$ in (A.2) as follows: Using the Hodge decomposition [18],

$$
\left\{\begin{aligned}
H^{q}(V, \mathrm{C}) & =H^{q, 0}(V) \oplus H^{q-1,1}(V) \oplus \cdots \oplus H^{0, q}(V), \\
& =H^{q-a, a}(V)=\overline{H^{a, q-a}(V)},
\end{aligned}\right.
$$

we define the integer $r_{2}$ by

$$
r_{2}=\min \left(r_{0}, s\right) \text { where } H^{a, q-a}(V)=0 \text { for } a>s .
$$

Then $r_{0} \leq q+1$, and this number measures how many terms vanish from the "outside" of the Hodge decomposition (A.3). Katz's refinement of the monodromy theorem is that

$$
\left(T^{N}-I\right)^{r} 2=0 .
$$

This improvement is of an analytic rather than a geometric nature.

4. Analytic proofs. Associated to the situation $f: X \rightarrow D$ there is a classifying space for Hodge structures (= period matrix domain) $\mathfrak{D}=G / H$ such that $T \in G$ and such that there is a holomorphic mapping

$$
D \stackrel{\phi}{\longrightarrow} D /\left\{T^{k}\right\}
$$

where $\phi(t)=\left\{\right.$ Hodge decomposition of $\left.H^{q}\left(V_{t}, \mathbf{C}\right)\right\}$ [14]. Passing to the universal covering $\mathcal{H}=\{z=x+\sqrt{-\bar{y}}, y>0\}$ of $D$, the period mapping (A.5) lifts to give

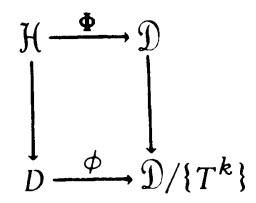

where $\Phi(z+1)=T \cdot \Phi(z)$. The mapping $\Phi$ is distance decreasing relative to the Poincaré metric on $\mathcal{H}$ and a suitable $G$-invariant metric on $\mathfrak{I}$. Using this, Borel (unpublished) gave a very simple proof that the eigenvalues of $T$ are roots of unity. Recently, this approach has been greatly extended by Schmid [17] who has given a complete asymptotic analys is of the Hodge decomposition $\phi(t)$ as $|t| \rightarrow 0$. As a by-product, Schmid obtains the exact position of $\log T$ in the Lie algebra of $G$ giving yet another proof of the strong monodromy theorem (A.4). In addition to this arithmetic property, Schmid finds that $T$ has very remarkable positivity properties, the simplest being that given by (13.5) on p. 266 of [14]. 
5. Computational questions. The problem of computing the P-L transformation in explicit examples is generally quite difficult. It seems to me that the global algorithmic procedure given by Landman in this paper is by far the best general method, as is perhaps suggested by the fairly complicated examples he is able to treat with relative ease. In particular, all of the P-L transformations which I have seen arising from the study of Feynman integrals in mathematical physics should be accessible to these techniques.

\section{SUPPLEMENTARY BIBLIOGRAPHY}

10. E. Brieskorn, Die monodromie der isolieters singularitäten von hyperflächen, Manuscripta Math. 29 (1970), 103-162.

11. C. H. Clemens, Picard-Lefschetz the orem for families of nonsingular algebraic varieties acquiring ordinary singularities, Trans. Amer. Math. Soc. 136 (1969), 93-108. MR $38 \# 2135$.

12. P. Deligne, Équations différentielles à points singuliers réguliers, Lecture Notes in Math., no. 163, Springer-Verlag, Berlin and New York, 1970.

13. P. Deligne and A. Grothendieck, S.G.A. seminars on monodromy, Inst. Hautes Études Sci., Paris (mineograph notes).

14. P. A. Griffiths, Periods of integrals on algebraic manifolds: Summary of main results and discussion of open problems, Bull. Amer。Math.Soc。 76 (1970), 228-296.

MR $41 \# 3470$ 。

15. N. Katz, Nilpotent connections and the monodromy theorem. Applications of a result of Turrittin, Inst. Hautes Études Sci. Publ. Math。39 (1970), 175-232.

16. J. Milnor, Singular points of complex hypersurfaces, Ann. of Math. Studies, no. 61, Princeton Univ。 Press, Princeton, N. J.; Univ。 of Tokyo Press, Tokyo, 1968. MR 39 \#969.

17. W. Schmid, Degeneration of Hodge structures (to appear).

18. A. Weil, Introduction à l'étude des variétés kahlériennes, Publ. Inst. Math. Univ. Nancago, VI, Actualités Sci. Indust., no. 1267, Hermann, Paris, 1958. MR 22 \#1921. 02912

DEPARTMENT OF MATHEMATICS, BROWN UNIVERSITY, PROVIDENCE, RHODE ISLAND 